\title{
Morphoelastic control of gastro-intestinal organogenesis: Theoretical predictions and numerical insights
}

\author{
V. Balbi ${ }^{\text {a }}$, E. Kuhl ${ }^{\mathrm{b}, \mathrm{c}, \mathrm{d}}$, P. Ciarletta ${ }^{\mathrm{a}, \mathrm{e}, *}$ \\ a CNRS and Sorbonne Universités, Université Paris 6, Institut Jean le Rond d'Alembert, UMR 7190, 4 place Jussieu case 162, 75005 Paris, France \\ ${ }^{\mathrm{b}}$ Department of Mechanical Engineering, Stanford University, 496 Lomita Mall, Stanford, CA 94305, USA

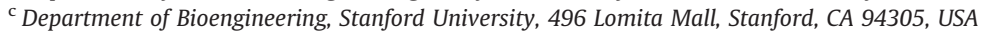 \\ d Department of Cardiothoracic Surgery, Stanford University, 496 Lomita Mall, Stanford, CA 94305, USA \\ ${ }^{\mathrm{e}}$ MOX, Politecnico di Milano and Fondazione CEN, piazza Leonardo da Vinci 32, 20133 Milano, Italy
}

\begin{abstract}
With nine meters in length, the gastrointestinal tract is not only our longest, but also our structurally most diverse organ. During embryonic development, it evolves as a bilayered tube with an inner endodermal lining and an outer mesodermal layer. Its inner surface displays a wide variety of morphological patterns, which are closely correlated to digestive function. However, the evolution of these intestinal patterns remains poorly understood. Here we show that geometric and mechanical factors can explain intestinal pattern for-mation. Using the nonlinear field theories of mechanics, we model surface morphogenesis as the instability problem of constrained differential growth. To allow for internal and external expansion, we model the gastrointestinal tract with homogeneous Neumann boundary conditions. To establish estimates for the folding pattern at the onset of folding, we perform a linear stability analysis supplemented by the perturbation theory. To predict pattern evolution in the post-buckling regime, we perform a series of nonlinear finite element simulations. Our model explains why longitudinal folds emerge in the esophagus with a thick and stiff outer layer, whereas circumferential folds emerge in the jejunum with a thinner and softer outer layer. In intermediate regions like the feline esophagus, longitudinal and circumferential folds emerge simultaneously. Our model could serve as a valuable tool to explain and predict alterations in esophageal morphology as a result of developmental disorders or certain digestive pathologies including food allergies.
\end{abstract}

\section{Introduction}

Since the pioneering experimental works of Hilton (1902) and Johnson (1910), it is well known that the functional structures of the gastrointestinal tract, such as villi, can emerge in vertebrates from the embryonic development of either plain circumferential folds or a more complex network of folds. The gastro-intestinal tissue originates from the embryonic differentiation of two primary matrices, the endoderm and the mesoderm. Later, the endoderm gives rise to the epithelium, while the mesoderm differentiates into several tissues including the mesenchyme and the smooth muscle layers (Yasugi,

\section{Article history:}

Received 11 December 2014 Received in revised form 12 February 2015

Accepted 22 February 2015

Available online 26 February 2015

\footnotetext{
* Corresponding author at: CNRS and Sorbonne Universités, Université Paris 6, Institut Jean le Rond d'Alembert, UMR 7190, 4 place Jussieu case 162, 75005 Paris, France.

E-mail address: pasquale.ciarletta@upmc.fr (P. Ciarletta).
} 
1993; McLin et al., 2009). A recent study on the development of intestine in vertebrates has shown that the morphogenesis of the intestinal mucosa is strictly related to the muscle layer differentiation in the gut (Shyer et al., 2013).

Fig. 1 illustrates the distinct folding patterns along the gastrointestinal tract varying from plain longitudinal folds in the esophagus via complex folding patterns in the stomach to circumferential folds in the intestine. In the chick, for instance, muscle layers form between days 8 and 16 after incubation, passing through three stages of differentiation. At day 8, a circumferentially oriented muscle forms, which corresponds to the onset of longitudinal ridges at the free surface of the epithelium. Around day 13, a second exterior muscular layer starts forming longitudinally and the longitudinal ridges transition to zigzag patterns is observed. Finally, at day 16 , a third longitudinally oriented muscle layer forms, interior to the first one, and simultaneously bulges arise from the zigzags, giving rise to the final villi. In the mouse gut, the muscle layer differentiates much faster than in the chick and develops in only 48 hours, without requiring the formation of an inner longitudinal layer; in the chick the same process takes 8 days. As a consequence, villi directly start forming from a bidimensional pattern at the luminal surface, without passing through folding and zigzags. The emergence of diverse mucosal structures in the embryonic intestines has recently attracted much interest from the mathematical and biomechanical communities; yet, the key mechanisms that determine such a wide variety of embryonic patterns remain elusive. Hannezo et al. proposed a simplified planar model of a single layered epithelium, lying on an elastic stroma, which reproduced villi and crypt formation in the small intestine and in the colon (Hannezo et al., 2011). In their fluid-like model of growth, cell proliferation induces a surface tension in the epithelium, and a buckling instability occurs once that a critical tension is reached.

Using a different constitutive approach based on the theory of thin plates, Nelson et al. proposed a two-dimensional model composed of a thin cell culture substrate lying between an upper cell monolayer and a lower elastic support. Similarly, the growth of the cell monolayer on the elastic support exerts stresses and can induce the buckling of the epithelium (Nelson et al., 2013).

Other approaches have considered intestinal tissues as nonlinear elastic bodies using the theory of volumetric growth (Rodriguez et al., 1994), which is based on the decomposition of the finite deformation tensor into a growth and an elastic component. Whenever the former introduces geometrical incompatibilities in the grown states, the latter must restore the continuity of matter and, as a consequence, gives rise to residual stresses inside the tissue (Skalak et al., 1996), which can eventually provoke a mechanical instability. Such a multiplicative decomposition has been successfully used in various morphoelastic models for studying pattern formation in soft materials (Ben Amar and Goriely, 2005) and living tissues (Li et al., 2012). Using this approach, recent work has attempted to study some of the instability patterns, which can occur at the inner surface of the gastro-intestinal mucosa. A number of simplified one-layered, cylindrical models have been formulated: Moulton and Goriely (2011) performed a linear stability analysis for studying the circumferential buckling of a growing cylinder under external pressure; Ciarletta and Ben Amar (2012a,b) proposed a variational method for studying the spatially constrained growth of thick-walled cylinder considering either circumferential or longitudinal folds. A great attention has been also payed to model the emergence of a more complex network of mucosal patterns, later developing villi and crypts in many vertebrates. Balbi and Ciarletta (2013) performed a linear stability analysis on growing onelayered thick-walled cylinders, showing that the initial geometry of the tissue can select a one-dimensional or bidimensional instability pattern, according to different villi formation mechanisms observed in several vertebrate species. Accounting for a differential growth between epithelium and mesenchyme, Ben Amar and Jia (2013) proposed a weakly nonlinear stability analysis for studying the emergence of the zigzag pattern, which typically develops in the chick embryo as a precursor of villi formation. Notwithstanding, such modeling approaches use simplifying geometrical assumptions, e.g., neglecting the heterogeneous two-layer setup. Most models do not take into account biologically relevant boundary conditions at the free boundaries, which should be stress-free. Moreover, the theoretical results can only give information on pattern selection around the instability threshold, whilst the later stages of embryonic mucosal pattern formation should be studied using a post-buckling numerical analysis. Few examples of post-buckling simulations in growth problems are present in the lit-erature. Li et al. (2011) studied the occurrence of wrinkling in a two-layered cylinder with fixed outer boundaries, modeling mucosal-submucosal differential volumetric growth. Papastavrou et al. (2013) studied the non-linear stability of the skin epithelium also including the finite element implementation of surface growth. Finally, Eskandari et al. (2013) investigated the role of volumetric growth in lung disease, where circumferential folding characterizes the pathologic state of the airway
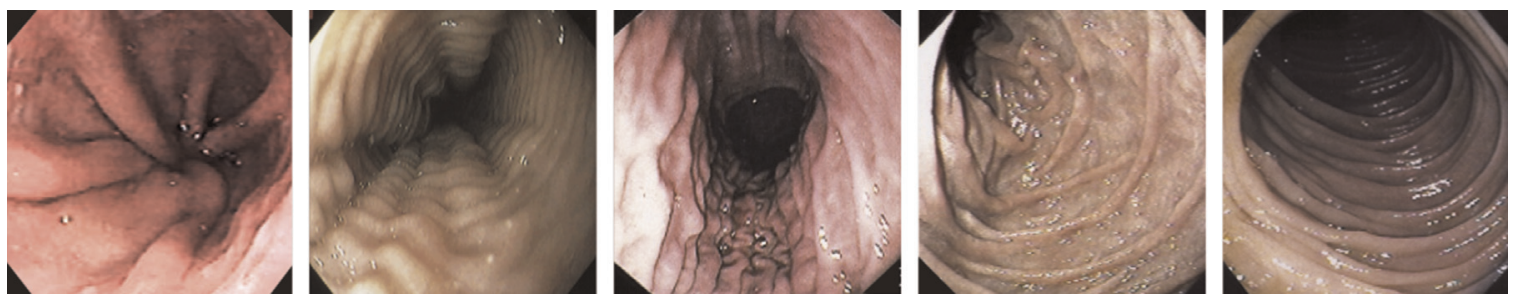

Fig. 1. Epithelial folding patterns in the human gut. Wavelengths and folding directions display a wide variety along the gastrointestinal tract: the esophagus displays pronounced longitudinal folding; the feline esophagus shows longitudinal folds overlaid with fine circumferential folds; the stomach displays a zigzag folding pattern; the valves of Kerckring in the duodenum fold primarily in the circumferential direction; the jejunum displays thin circumferential folds; from left to right, adopted with permission from Wilcox et al. (2012). 
mucosa.

All these models assume that the muscle surrounding the epithelium-mesenchyme tissue is rigid, implying that the tissue cannot move outward while it grows. This assumption allows a stable implementation of non-linear numerical methods to solve the elastic equilibrium problem; yet, it is non-physiological, since the non-zero displacements at the outer surface of the epithelial-mesenchymal interface may critically influence pattern selection.

In this work, we aim at modeling the occurrence of both the one-dimensional and the bi-dimensional mucosal patterns observed during intestinal development in different vertebrate species. In Section 2, we introduce a finite elastic model describing the embryonic intestine as a two-layered incompressible cylinder. Using the theory of finite growth, we account for differential growth between endoderm and mesoderm, using a non-linear elastic constitutive model for the incompressible tissue. In Section 3, we perform a linear stability analysis following the method of incremental deformations superposed on finite strains and derive the differential growth thresholds for the onset of the instability. In Section 4, we perform fully nonlinear simulations, using a user-defined material subroutine for growth implemented in a commercial finite element software, for both validating the results of the linear stability analysis and investigating the morphological evolution of the mucosal patterns in the nonlinear regime. Our results are reported in Section 5 and finally discussed in Section 6 with respect to the available experimental data in the biological literature.

\section{The morphoelastic model: kinematic and constitutive assumptions}

We model the embryonic intestine as a two-layered cylinder. The inner epithelial layer derives from the primary endodermal matrix and the outer layer includes the mesenchyme and the muscles which both originate from the primary mesodermal matrix.

\subsection{Kinematics}

Let $\mathcal{E} \subset \mathbb{R}^{3}$ be the three-dimensional Euclidean space, so that $\mathcal{B}_{0}, \mathcal{B}_{a} \subset \mathcal{E}$ are two regions occupied by the cylinder at two different instants of time. Let $\mathcal{B}_{0}$ be the reference configuration with orthonormal basis $\left\{\mathbf{E}_{R}, \mathbf{E}_{\Theta}, \mathbf{E}_{Z}\right\}$ and $\mathcal{B}_{a}$ the spatial or deformed configuration at time $t$, with orthonormal basis $\left\{\mathbf{e}_{r}, \mathbf{e}_{\theta}, \mathbf{e}_{z}\right\}$. We name $R_{i}$ and $R_{0}$, the initial internal and external radii, respectively. Let $R_{m}$ be the initial contact radius at the interface of the two layers and $L$ the initial length of the tube. We denote the internal, the contact, and the external radii as $r_{i}, r_{m}$ and $r_{0}$, respectively, and the length of the tube in the deformed state as $l$. Fig. 2 depicts the geometric representation of the model.

The growth process of the intestinal wall is modeled as a deformation $\chi$ from the reference configuration $\mathcal{B}_{0}$ to the spatial configuration $\mathcal{B}_{a}$, defined as

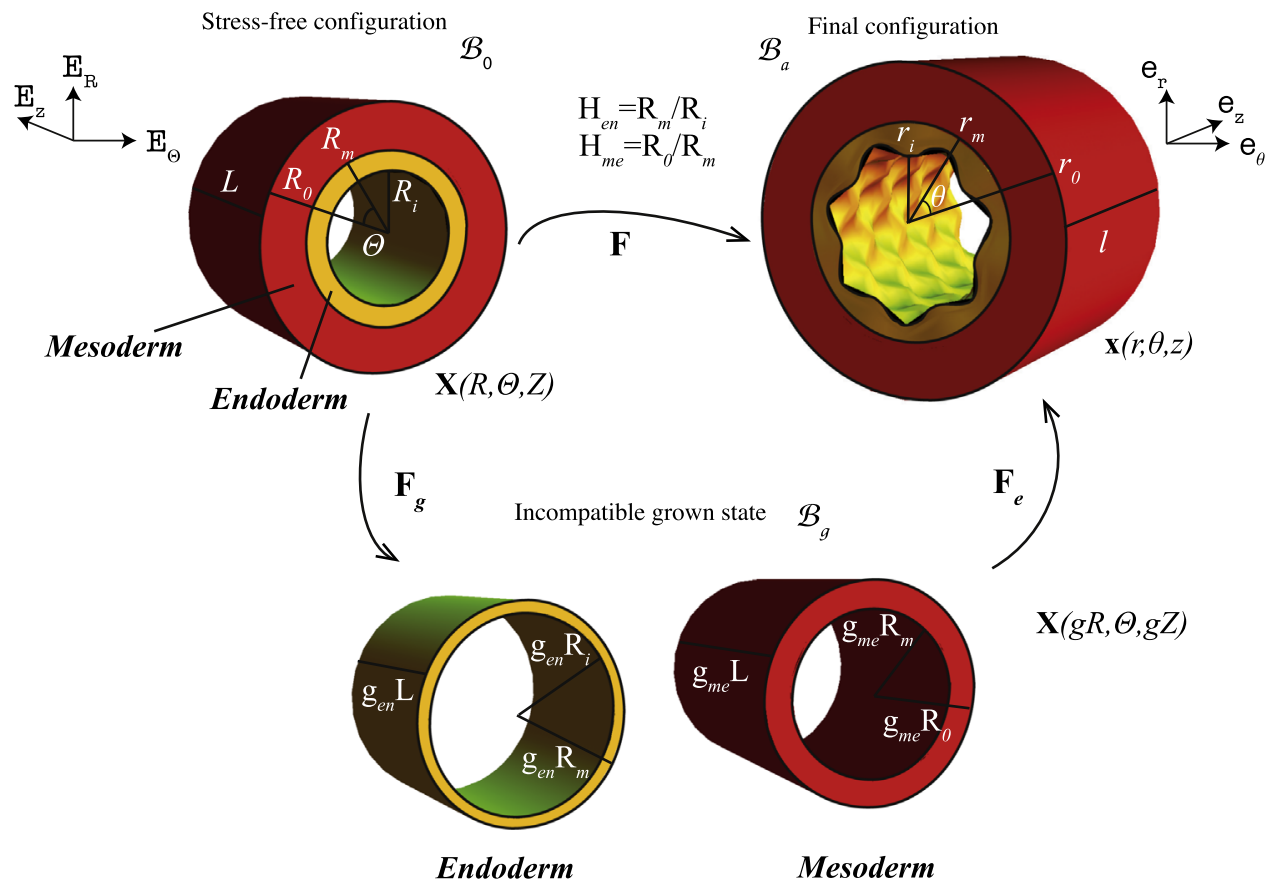

Fig. 2. Kinematics of the two-layered embryonic gut and representation of the deformation $\chi$ from the stress free reference configuration $\mathcal{B}_{0}$ to the deformed residually stressed configuration $\mathcal{B}_{a}$, with the use of cylindrical coordinates. 


$$
\chi: \mathcal{B}_{0} \rightarrow \mathcal{B}_{a}, \quad \mathbf{X}=\chi(\mathbf{X})
$$

where $\mathbf{X}$ and $\mathbf{X}$ are the material and spatial position vectors respectively. As first proposed by Rodriguez et al. (1994), the deformation gradient $\mathbf{F}=\partial \mathbf{x} / \partial \mathbf{X}$ associated with the deformation in Eq. (1) can be split into a growth and an elastic component, $\mathbf{F}_{g}$ and $\mathbf{F}_{e}$, so that the following relation holds:

$$
\mathbf{F}=\mathbf{F}_{e} \mathbf{F}_{g}
$$

Since the cellular components of the embryonic tissue are mostly composed of water, we assume the intestine to be incompressible. The incompressibility condition reads

$$
\operatorname{det} \mathbf{F}_{e}=1 \text {. }
$$

Furthermore, we consider a homogeneous, isotropic growth so that the growth tensor $\mathbf{F}_{g}$ in Eq. (2) has the following form:

$$
\mathbf{F}_{g}=g \mathbf{I}
$$

where $g$ denotes the volumetric growth factor of each layer and $\mathbf{I}$ is the identity tensor. Furthermore we assume that no tractions are acting on the internal and external surfaces. In the following section, the constitutive relations which govern the mechanical behavior of the intestinal wall are presented.

\subsection{Constitutive relations}

The intestine is modeled as hyperelastic, isotropic, incompressible, two-layered tube. We recall that the right and left elastic Cauchy-Green tensors are given by $\mathbf{C}_{e}=\mathbf{F}_{e}^{T} \mathbf{F}_{e}$ and $\mathbf{B}_{e}=\mathbf{F}_{e} \mathbf{F}_{e}^{T}$ respectively. Hence the elastic strain energy function of each layer can be defined as

$$
\psi\left(\mathbf{F}_{e}\right)=\frac{1}{2} \mu\left(\operatorname{tr} \mathbf{C}_{e}-3\right)-p\left(\operatorname{det} \mathbf{F}_{e}-1\right)
$$

where $\mu$ is the shear modulus, tr is the trace operator, and $p$ is the Lagrange multiplier in the form of the hydrostatic pressure to locally enforce the incompressibility condition. From Eq. (5) the nominal stress can be calculated as

$$
\mathbf{S}=\frac{\partial \psi\left(\mathbf{F}_{e}\right)}{\partial \mathbf{F}_{e}}-p \mathbf{F}_{e}^{-1}=\mu \mathbf{F}_{e}^{T}-p \mathbf{F}_{e}^{-1},
$$

and its spatial counterpart, the Cauchy stress $\sigma=\mathbf{F}_{e} \mathbf{S}$ follows as

$$
\boldsymbol{\sigma}=\mu \mathbf{B}_{e}-p \mathbf{I}
$$

In the absence of body forces, the balance of linear momentum reduces to

$$
\operatorname{Div} \mathbf{S}=\mathbf{0} \text { in } \mathcal{B}_{0} \text { and } \operatorname{div} \boldsymbol{\sigma}=\mathbf{0} \text { in } \mathcal{B}_{t},
$$

where Div and div are the divergence operators in the material and spatial configurations, respectively. Moreover, the traction-free boundary conditions at the internal and external surfaces read

$$
\begin{aligned}
& \mathbf{N} \cdot \mathbf{S}=\mathbf{0} \text { on } R: R=R_{i} \quad \text { and } \quad \mathbf{n} \cdot \boldsymbol{\sigma}=\mathbf{0} \text { on } r: r=r_{i}, \\
& \mathbf{N} \cdot \mathbf{S}=\mathbf{0} \text { on } R: R=R_{0} \quad \text { and } \quad \mathbf{n} \cdot \boldsymbol{\sigma}=\mathbf{0} \text { on } r: r=r_{0},
\end{aligned}
$$

where $\partial \mathcal{B}_{0}=\left\{R: R=R_{i}\right\} \cup\left\{R: R=R_{0}\right\}$ and $\partial \mathcal{B}_{a}=\left\{r: r=r_{i}\right\} \cup\left\{r: r=r_{0}\right\}$ denote the initial and grown boundaries of the inner and outer surfaces.

\section{Linear stability analysis}

We are interested in studying the instability patterns, which emerge at the internal surface of the intestinal wall induced by volumetric growth. Therefore, we perform a linear stability analysis using a method provided by the perturbation theory. First, we consider the solution of the elastic problem in the form of a perturbation series expanded to the first order. We solve the equilibrium problem in Eqs. (8) and (9) at the zeroth order to calculate the basic axial-symmetric solution. Then we consider the first order correction to the basic solution in the form of a small perturbation and solve the associated perturbed equilibrium problem.

\subsection{Basic axial-symmetric solution}

In this section we derive the zeroth-order solution of the equilibrium problem in Eqs. (8) and (9). First, let us assume a basic axial-symmetric deformation of the following form: 


$$
\begin{aligned}
& r(R, \Theta, Z)=\sqrt{g_{l}^{3} R^{2}+a_{l}} \\
& \theta(R, \Theta, Z)=\Theta \\
& z(R, \Theta, Z)=Z,
\end{aligned}
$$

where $l=\{e n, m e\}$ such that $g_{e n}$ and $g_{m e}$ are the volumetric growth factors of the endodermal and mesodermal layers and $a_{e n}=\left(r_{i}^{2}-g_{e n}^{3} R_{i}^{2}\right)$ and $a_{m e}=\left(r_{m}^{2}-g_{m e}^{3} R_{m}^{2}\right)$ follow from the global incompressibility condition. For the sake of notation simplicity, unless explicitly stated, we will from now on omit the sub- and superscript $l$, which refers to the individual layers. The elastic contribution to the deformation gradient in Eq. (2) reads

$$
\mathbf{F}_{e}=\operatorname{diag}\left(\lambda_{r}(r), \lambda_{\theta}(r), \lambda_{z}\right),
$$

where

$$
\lambda_{r}(r)=g R / r \quad \text { and } \quad \lambda_{\theta}(r)=r /(g R) \quad \text { and } \quad \lambda_{z}=1 / g
$$

are the principal stretches. From Eq. (7), we conclude that the only non-zero components of the Cauchy stress are the principal stresses:

$$
\begin{aligned}
& \sigma_{r r}(r)=\mu \lambda_{r}^{2}(r)-p(r) \\
& \sigma_{\theta \theta}(r)=\mu \lambda_{\theta}^{2}(r)-p(r) \\
& \sigma_{z z}(r)=\mu \lambda_{z}^{2}-p(r),
\end{aligned}
$$

where $p(r)$ is the Lagrange multiplier, which takes the interpretation of the hydrostatic pressure. With Eqs. (13), the equilibrium equations $(8)$ reduce to

$$
\frac{d \sigma_{r r}(r)}{d r}+\frac{\sigma_{r r}(r)-\sigma_{\theta \theta}(r)}{r}=0
$$

with the boundary conditions:

$$
\sigma_{r r}^{e n}(r)=0 \text { on } r=r_{i} \quad \text { and } \quad \sigma_{r r}^{m e}(r)=0 \text { on } r=r_{0} .
$$

To ensure continuity of the radial component of the Cauchy stress at the contact interface, we also require that

$$
\sigma_{r r}^{e n}\left(r_{m}^{e n}\right)=\sigma_{r r}^{m e}\left(r_{m}^{m e}\right)
$$

The Lagrange multipliers in Eqs. (13) are calculated by integrating Eq. (8) with the boundary conditions in Eq. (15), as follows:

$$
\begin{aligned}
& p_{e n}(r)=\mu_{e n} \lambda_{r}^{2}(r)+\mu_{e n} \int_{r_{i}}^{r} \frac{\lambda_{r}^{2}(s)-\lambda_{\theta}^{2}(s)}{s} d s \\
& p_{m e}(r)=\mu_{m e} \lambda_{r}^{2}(r)-\mu_{m e} \int_{r}^{10} \frac{\lambda_{r}^{2}(s)-\lambda_{\theta}^{2}(s)}{s} d s,
\end{aligned}
$$

where the internal and external radii follow with (3) and (10):

$$
r_{i}=\sqrt{\left(\frac{g_{e n}}{g_{m e}}\right)^{3}\left(\frac{1}{H_{e n}^{2}}-\frac{1}{H_{m e}^{2}}\right)+r_{m}^{2}} \text { and } r_{0}=\sqrt{1-\frac{1}{H_{m e}^{2}}+r_{m}^{2}} .
$$

Here $H_{e n}=R_{m} / R_{i}$ and $H_{m e}=R_{0} / R_{m}$ denote the geometric aspect ratios of the internal and external layers, respectively. Using the continuity condition in Eq. (16), we solve Eq. (14) with the boundary conditions in Eq. (15):

$$
\begin{aligned}
& \frac{g_{m e}}{H_{m e}^{2}}\left(\frac{1}{r_{m}^{2}}-\frac{H_{m e}^{4}}{H_{m e}^{2}\left(1+r_{m}^{2}\right)-1}\right)+\frac{\mu_{m e}}{\mu_{e n}} \frac{g_{e n}^{4}}{g_{m e}^{3}}\left(\frac{1}{\left(\frac{g_{e n}}{g_{m e}}\right)^{3}\left(1-H_{e n}^{2}\right)+H_{e n}^{2} H_{m e}^{2} r_{m}^{2}}-\frac{1}{H_{m e}^{2} r_{m}^{2}}\right) \\
& +g_{m e} \log \left(\frac{r_{m}\left(H_{m e}^{2}\left(r_{m}^{2}+1\right)-1\right)}{H_{m e}^{4}}\right)-g_{e n} \frac{\mu_{m e}}{\mu_{e n}} \log \left(\frac{\left(\frac{g_{e n}}{g_{m e}}\right)^{3}\left(1-H_{e n}^{2}\right)+r_{m}^{2}\left(H_{e n}^{2} H_{m e}^{2}\right)}{r_{m}^{2}}\right)=0 .
\end{aligned}
$$



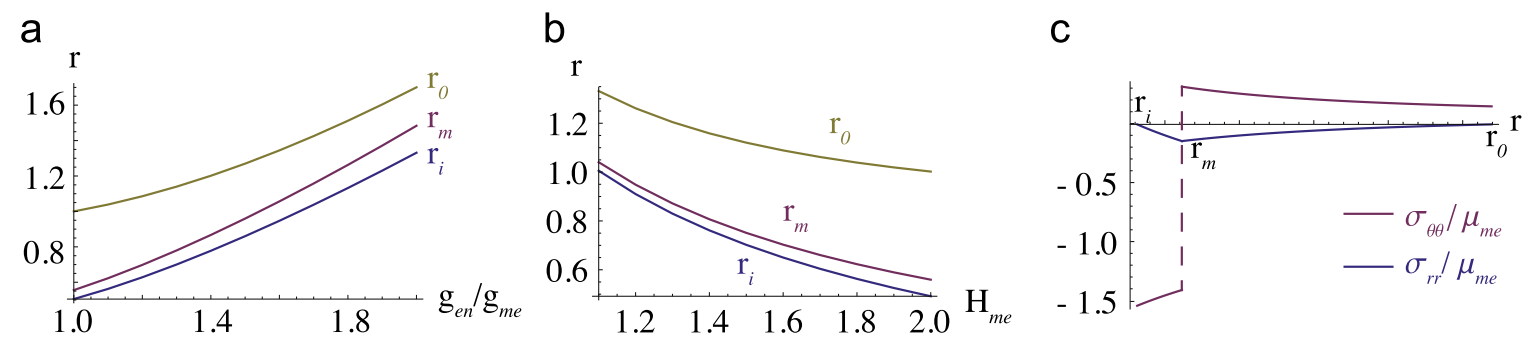

Fig. 3. Deformed internal, external, and contact radii, $r_{i}, r_{0}, r_{m}$ as functions of the growth ratio $g_{\text {en }} / g_{m e}$ at fixed $H_{e n}=1.1$ and $H_{m e}=1.8$ (a), and as a function of the mesoderm aspect ratio $H_{m e}$, at fixed $H_{e n}=1.1$ (b), and $g_{e n} / g_{m e}=1.1$, setting $\mu_{m} d \mu_{e n}=10$. Radial and circumferential components of the Cauchy stress $\sigma$ plotted with respect to $r$ at fixed $g_{e} g_{m e}=1.1$ with $g_{m e}=1, \mu_{m} k \mu_{e n}=10, R_{0}=1, H_{e n}=1.1$ and $H_{m e}=1.8$ (c).

Finally, we calculate the deformed radius at the interface between the two layers $r_{m}$ by numerically solving Eq. (19) with an explicit Newton method. In Fig. 3, the deformed internal, external, and contact radii, $r_{i}, r_{0}, r_{m}$ are plotted against $H_{e n}(\mathrm{a})$ and $H_{m e}$ (b), and the radial and circumferential components of the Cauchy stress in Eq. (7) are depicted as a function of $r$.

\subsection{Incremental deformations}

In this section, we consider the first order correction of the basic solution calculated in Section 3.1. For this purpose, we will use the elastic theory of incremental deformations superposed on finite displacements, employing the derivations and the notation from Haughton and Ogden (1979) and Ogden (1997). Let $\chi^{1}(\mathbf{x})$ be an incremental deformation, superposed on the axis-symmetric solution, so that the perturbed mapping reads

$$
\bar{\chi}(\mathbf{X})=\chi(\mathbf{X})+\varepsilon \chi^{1}(\mathbf{x}),
$$

where $\varepsilon$ is an arbitrary small parameter. To perform a linear stability analysis, we solve the perturbed equilibrium problem associated to the deformation in Eq. (20) and calculate the instability thresholds and the associated critical modes in terms of the volumetric growth factors $g_{e n}$ and $g_{m}$. The incremental deformation $\chi^{1}$ can be expressed as

$$
\chi^{1}(r, \theta, z)=u(r, \theta, z) \mathbf{e}_{r}+v(r, \theta, z) \mathbf{e}_{\theta}+w(r, \theta, z) \mathbf{e}_{z},
$$

where $u, v, w$ are three scalar functions. The elastic deformation gradient associated with the deformation in Eq. (20) takes the following form:

$$
\overline{\mathbf{F}}_{e}=\mathbf{F}_{e}+\varepsilon \boldsymbol{\Gamma} \dot{\mathbf{F}}_{e},
$$

where $\mathbf{\Gamma}=\partial \chi^{1}(\mathbf{x}) / \partial \mathbf{x}$ is the spatial displacement gradient:

$$
\boldsymbol{\Gamma}=\left(\begin{array}{ccc}
u_{, r} & \left(u_{, \theta}-v\right) / r & u_{, z} \\
v_{, r} & \left(v_{, \theta}+u\right) / r & v_{, z} \\
w_{, r} & \left(v_{, \theta}\right) / r & w_{, z}
\end{array}\right),
$$

and the comma denotes the partial derivative. After the perturbation, the nominal stress tensor reads

$$
\overline{\mathbf{S}}=\mathbf{S}+\varepsilon \dot{\mathbf{S}},
$$

where $S$ is the nominal stress in Eq. (6) and $\dot{S}$ is the first order increment of $S$, which follows from differentiating Eq. (6). The spatial counterpart of the nominal stress in Eq. (24) can be calculated as $\overline{\mathbf{S}}_{0}=\mathbf{F}_{e} \overline{\mathbf{S}}=\mathbf{S}_{0}+\varepsilon \dot{\mathbf{S}}_{0}$, where $\mathbf{S}_{0}=\mathbf{F}_{e} \mathbf{S}$, and $\dot{\mathbf{S}}_{0}$ is given by

$$
\dot{\mathbf{S}}_{0}=\mathbf{F}_{e} \dot{\mathbf{S}}=\mathcal{A}_{0}^{1} \boldsymbol{\Gamma}+p \boldsymbol{\Gamma}-q \mathbf{I} .
$$

In Eq. (25), we have introduced the increment $q$ of the Lagrange multiplier $p$ and the fourth order tensor of instantaneous moduli $\mathcal{A}_{0}^{1}$, which in components, reads

$$
\mathcal{A}_{0 h k l j}^{1}=F_{h l} F_{l \beta} \frac{\partial^{2} \psi}{\partial F_{k_{l}} \partial F_{j \beta}}=\mu \delta_{k j} B_{h l},
$$

where $\delta_{k j}$ is the Kronecker delta. Once we have derived the incremental quantities, we can write the incremental counterpart of the equilibrium equations in Eq. (8), which reads

$$
\operatorname{div} \dot{\mathbf{S}}_{0}=\mathbf{0},
$$

where $\dot{\mathbf{S}}_{0}$ is given by Eq. (25). The boundary conditions in Eq. (9) take the following form: 


$$
\begin{array}{ll}
\mathbf{n} \cdot \dot{\mathbf{S}}_{0}^{e n}=\mathbf{0} & \text { on } r: r=r_{i} \\
\mathbf{n} \cdot \dot{\mathbf{S}}_{0}^{m e}=\mathbf{0} & \text { on } r: r=r_{0}
\end{array}
$$

and the incompressibility condition in Eq. (3) reads

$$
\operatorname{tr} \Gamma=0 \text {. }
$$

In conclusion, Eq. (27) reduces to the following set of three partial differential equations:

$$
\begin{aligned}
& \frac{\partial\left(r \dot{S}_{0 r r}\right)}{\partial r}+\frac{\partial \dot{S}_{0 \theta r}}{\partial \theta}+r \frac{\partial \dot{S}_{0 z r}}{\partial z}-\dot{S}_{0 \theta \theta}=0 \\
& \frac{\partial\left(r \dot{S}_{0 r \theta}\right)}{\partial r}+\frac{\partial \dot{S}_{0 \theta \theta}}{\partial \theta}+r \frac{\partial \dot{S}_{0 z \theta}}{\partial z}+\dot{S}_{0 \theta r}=0 \\
& \frac{\partial\left(r \dot{S}_{0 r z}\right)}{\partial r}+\frac{\partial \dot{S}_{0 \theta z}}{\partial \theta}+r \frac{\partial \dot{S}_{0 z z}}{\partial z}=0 .
\end{aligned}
$$

It follows from Eq. (23) that the incremental incompressibility condition in Eq. (29) reduces to

$$
u_{, r}+\frac{u+v_{, \theta}}{r}+w_{, z}=0
$$

In the following section we will describe the formalism we used to numerically solve the incremental equilibrium problem in Eqs. (30), together with Eq. (31) and the boundary conditions in Eq. (28).

\subsection{Stroh formulation}

In this section we use a formalism originally developed by Stroh (1962) for steady state elastic problem, and later applied to pre-stressed elastic media (Chadwick, 1997). The Stroh formalism allows us to transform the set of four partial differential equations in Eqs. (30) and (31) with the boundary conditions in Eq. (28), into a set of six ordinary differential equations of first order with initial conditions for both layers, $l=\{e n, m e\}$. Using the optimal, Hamiltonian form based on the Stroh formalism Fu (2007) allows great simplifications in solving a large variety of problems in general anisotropic elasticity (see Destrade et al., 2009 for a more detailed discussion on numerical issues). First let us consider that the separation of variables, so that we can express the components in Eq. (21) and the increment of the Lagrange multiplier in Eq. (25) in the following form:

$$
\begin{aligned}
& u=U(r) \cos (m \theta) \cos \left(k_{z} z\right) \\
& v=V(r) \sin (m \theta) \cos \left(k_{z} z\right) \\
& w=W(r) \cos (m \theta) \sin \left(k_{z} z\right) \\
& q=Q(r) \cos (m \theta) \cos \left(k_{z} z\right),
\end{aligned}
$$

where $m$ and $k_{z}=2 \pi n / L$ (with $m, n \in \mathbb{N}$ ) are the circumferential and longitudinal modes respectively, and $U(r), V(r), W(r), Q(r)$ are four scalar functions. Similarly, we write the incremental stress components $\dot{S}_{0 r r}, \dot{S}_{0 r \theta}, \dot{S}_{0 r z}$ as follows:

$$
\begin{aligned}
& \dot{S}_{0 r r}=s_{0 r r}(r) \cos (m \theta) \cos \left(k_{z} z\right) \\
& \dot{S}_{0 r \theta}=s_{0 r \theta}(r) \sin (m \theta) \cos \left(k_{z} z\right) \\
& \dot{S}_{0 r z}=s_{0 r z}(r) \cos (m \theta) \sin \left(k_{z} z\right),
\end{aligned}
$$

where $s_{0 r r}(r), s_{0 r \theta}(r), s_{0 r z}(r)$ are three scalar functions. Substituting Eqs. (32) and (33) into Eqs. (31) and (25) gives

$$
U^{\prime}=-\frac{U+m V}{r}-k_{z} W, \quad V^{\prime}=\frac{s_{0 \mathrm{r} \theta}+m U p+V p}{\mathcal{A}_{0 \mathrm{r} \theta \mathrm{r} \theta}^{1} r}, \quad W^{\prime}=\frac{s_{0 r z}+r k_{z} U p}{A_{0 \mathrm{rzrz}}^{1} r},
$$

where' denotes the derivative with respect to the radial coordinate $r$. Similarly, substituting Eq. (33) into Eq. (25) yields the function $Q$ :

$$
Q=-\frac{s_{0 r r}}{r}+\left(A_{0 r r r}^{1}+p\right) U^{\prime}
$$

Finally, by substituting Eqs. (32) and (33) into the three incremental equilibrium equations (30), we obtain the following set of equations: 


$$
\begin{aligned}
& \left(A_{0 \theta \theta \theta \theta}^{1}+A_{0 \theta r \theta r}^{1} m^{2}+p+A_{0 z r z r}^{1} k_{z}^{2} r^{2}\right) U+m\left(A_{0 \theta r \theta r}^{1}+A_{0 \theta \theta \theta \theta}^{1}+p\right) V+s_{0 r r}-r\left(\left(A_{0 r r r r}^{1}+p\right) U^{\prime}+p\left(m V^{\prime}+k_{z} r W^{\prime}\right)+s_{0 r r}^{\prime}\right)=0, \\
& m\left(A_{0 \theta r \theta r}^{1}+A_{0 \theta \theta \theta \theta}^{1}+p\right) U+\left(A_{0 \theta r \theta r}^{1}+m^{2}\left(A_{0 \theta \theta \theta \theta}^{1}+p\right)+A_{0 z \theta z \theta}^{1} k_{z}^{2} r^{2}\right) V+\left(m k_{z} r p\right) W \\
& +m s_{0 r r}-m r\left(A_{0 r r r r}^{1}+p\right) U^{\prime}-p r V^{\prime}-r s_{0 r \theta}^{\prime}=0 \\
& k_{z} r s_{0 r r}-\left(k_{z} m r p\right) V+\left(A_{0 \theta z z z}^{1} m^{2}-k_{z}^{2} r^{2}\left(A_{0 z z z z}^{1}+p\right)\right) W-k_{z}\left(A_{0 r r r r}^{1}+p\right) r^{2} U^{\prime}+r s_{0 r z}^{\prime}=0
\end{aligned}
$$

In order to write the set of six first order ordinary differential equations from (34) and (36) in compact form, we introduce the displacement-traction vector $\boldsymbol{\eta}$ :

$$
\boldsymbol{\eta}(r)=\left[\begin{array}{c}
\mathbf{U}(r) \\
r \mathbf{S}(r)
\end{array}\right] \text { with } \begin{aligned}
& \mathbf{U}(r)=[U(r), V(r), W(r)]^{T} \\
& \mathbf{S}(r)=\left[s_{0 r r}(r), s_{0 r \theta}(r), s_{0 r z}(r)\right]^{T} .
\end{aligned}
$$

Now, we can rewrite Eqs. (34) and (36) in the following format:

$$
\frac{d \boldsymbol{\eta}(r)}{d r}=\frac{1}{r} \mathbf{G}(r) \boldsymbol{\eta}(r),
$$

where $\mathbf{G}$ is the so-called Stroh matrix, which takes the following block form:

$$
\mathbf{G}=\left(\begin{array}{ll}
\mathbf{G}_{1} & \mathbf{G}_{2} \\
\mathbf{G}_{3} & \mathbf{G}_{4}
\end{array}\right) .
$$

The four blocks in Eq. (39) are given by

$$
\begin{aligned}
& \mathbf{G}_{1}=\left(\begin{array}{ccc}
-1 & -m & -k_{z} r \\
m_{\sigma_{1}} & \sigma_{1} & 0 \\
k_{z} r_{2} & 0 & 0
\end{array}\right), \quad \mathbf{G}_{2}=\left(\begin{array}{ccc}
0 & 0 & 0 \\
0 & 1 / \alpha_{1} & 0 \\
0 & 0 & 1 / \alpha_{2}
\end{array}\right) \\
& \mathbf{G}_{3}=\left(\begin{array}{lll}
\kappa_{11} & \kappa_{12} & \kappa_{13} \\
\kappa_{12} & \kappa_{22} & \kappa_{23} \\
\kappa_{13} & \kappa_{23} & \kappa_{33}
\end{array}\right), \quad \mathbf{G}_{4}=\left(\begin{array}{ccc}
1 & -m_{\sigma_{1}} & -k_{z} r_{\sigma_{2}} \\
m & -\sigma_{1} & 0 \\
k_{z} r & 0 & 0
\end{array}\right)
\end{aligned}
$$

with $\mathbf{G}_{4}=-\mathbf{G}_{1}^{T}$ and

$$
\begin{aligned}
& \kappa_{11}=m^{2}\left(\nu_{1}-\alpha_{1} \sigma_{1}^{2}\right)+k_{z}^{2} r^{2}\left(\nu_{2}-\alpha_{2} \sigma_{2}^{2}\right)+\mathcal{A}_{0 r r r}^{1}+\mathcal{A}_{0 \theta \theta \theta \theta}^{1}+2 \alpha_{1} \sigma_{1} \\
& \kappa_{12}=m\left(\mathcal{A}_{0 r r r r}^{1}+\mathcal{A}_{0 \theta \theta \theta \theta}^{1}+2 \alpha_{1} \sigma_{1 l}+\nu_{1}-\alpha_{1} \sigma_{1}^{2}\right) \\
& \kappa_{13}=k_{z} r\left(\mathcal{A}_{0 r r r r}^{1}+\alpha_{1} \sigma_{1}\right) \\
& \kappa_{22}=m^{2}\left[\mathcal{A}_{0 r r r}^{1}+\mathcal{A}_{0 \theta \theta \theta \theta}^{1}+2 \alpha_{1} \sigma_{1}\right]+k_{z}^{2} r^{2} \nu_{3}+\nu_{1}-\alpha_{1} \sigma_{1}^{2} \\
& \kappa_{23}=m k_{z} r\left(\mathcal{A}_{0 r r r r}^{1}+2 \alpha_{1} \sigma_{1}\right) \\
& \kappa_{33}=m^{2} \alpha_{3}+k_{z}^{2} r^{2}\left(\mathcal{A}_{0 r r r r}^{1}+\mathcal{A}_{0 z z z z}^{1}+2 \alpha_{3} \sigma_{3}\right),
\end{aligned}
$$

and

$$
\begin{aligned}
& \nu_{1}=A_{0 \theta r \theta r}^{1}, \quad \alpha_{1}=\mathcal{A}_{0 \mathrm{rer} \theta}^{1}, \quad \sigma_{1}=p / \alpha_{1}, \\
& \nu_{2}=A_{0 z r r r}^{1}, \quad \alpha_{2}=\mathcal{A}_{0 \text { rrzz }}^{1}, \quad \sigma_{2}=p / \alpha_{2} \\
& \nu_{3}=A_{0 z \theta z \theta}^{1}, \quad \alpha_{3}=\mathcal{A}_{0 \theta z \theta z}^{1}, \quad \sigma_{3}=p / \alpha_{3} .
\end{aligned}
$$

In the next section, we introduce the surface impedance method, which allows us to numerically find a solution of Eq. (38).

\subsection{The surface impedance method}

The surface impedance method is largely used for studying wave propagation in inhomogeneous solids. It was first introduced by Biryukov (1985) and developed for cylindrical geometries by Norris and Shuvalov (2010). Here we use this method to implement a fast and efficient numerical procedure to numerically solve Eq. (38). First, we define a set of independent solutions $\boldsymbol{\eta}_{n}, \nexists\{1, \ldots, 6\}$, of the system in Eq. (38). Then we introduce the $6 \times 6$ matricant $\mathbf{M}\left(r, r_{k}\right)$ as the following block matrix for the inner and outer layers, $k=(i, o)$ :

$$
\mathbf{M}\left(r, r_{k}\right)=\left(\begin{array}{ll}
\mathbf{M}_{1}\left(r, r_{k}\right) & \mathbf{M}_{2}\left(r, r_{k}\right) \\
\mathbf{M}_{3}\left(r, r_{k}\right) & \mathbf{M}_{4}\left(r, r_{k}\right)
\end{array}\right)=\mathcal{N}(r) \mathcal{N}^{-1}\left(r_{k}\right),
$$


where $\mathcal{N}$ is the integral matrix, defined as

$$
\mathcal{N}(r)=\left[\boldsymbol{\eta}_{1}, \ldots, \boldsymbol{\eta}_{6}\right]
$$

The matricant in Eq. (43) is the solution of the initial value problem for the inner and outer layers, $k=(i, o)$ :

$$
\frac{d \mathbf{M}}{d r}\left(r, r_{k}\right)=\frac{1}{r} \mathbf{G}(r) \mathbf{M}\left(r, r_{k}\right) \text { with } \mathbf{M}\left(r_{k}, r_{k}\right)=\mathbf{I}_{(6)}
$$

where $\mathbf{I}_{(6)}$ is the $6 \times 6$ identity matrix. Now, we define the conditional impedance matrix $\mathbf{z}=\mathbf{z}\left(r, r_{k}\right)$ as the $3 \times 3$ matrix, such that

$$
r \mathbf{S}=\mathbf{z} \mathbf{U}
$$

The form of the impedance matrix in Eq. (46), depends on the boundary condition at $r=r_{k}$. For a zero traction condition at $r=r_{k}, \mathbf{z}$ can be expressed in the following form:

$$
\mathbf{z}=\mathbf{M}_{3} \mathbf{M}_{1}^{-1} .
$$

Substituting Eqs. (37) and (46) in Eq. (38), we obtain

$$
\begin{aligned}
& \frac{d}{d r} \mathbf{U}=\frac{1}{r} \mathbf{G}_{1} \mathbf{U}+\frac{1}{r} \mathbf{G}_{2} \mathbf{z} \mathbf{U} \\
& \frac{d}{d r}(\mathbf{z} \mathbf{U})=\frac{1}{r} \mathbf{G}_{3} \mathbf{U}-\frac{1}{r} \mathbf{G}_{1}^{T} \mathbf{z} \mathbf{U}
\end{aligned}
$$

and substituting Eq. (48).1 into Eq. (48).2 we derive the differential matrix Riccati equation:

$$
\frac{d \mathbf{z}}{d r}=\frac{1}{r}\left(\mathbf{G}_{3}-\mathbf{G}_{1}^{T} \mathbf{z}-\mathbf{z G}_{1}-\mathbf{z G}_{2} \mathbf{z}\right) .
$$

From Eq. (46), the initial condition of zero traction at the inner surface $r_{i}$ rewrites

$$
\mathbf{z}^{e n}\left(r_{i}\right)=\mathbf{0},
$$

and the continuity condition at the interface of the two layers reads

$$
\mathbf{z}^{e n}\left(r_{m}^{e n}\right)=\mathbf{z}^{m e}\left(r_{m}^{m e}\right)
$$

Using the first of Eq. (50) as the initial condition, we integrate the Riccati equation (49) for the endoderm, from $r=r_{i}$ to $r=r_{m}^{e n}$. Using this solution and the continuity condition in Eq. (51) we derive the initial condition for the mesoderm at $r=r_{m}^{m e}$ and we integrate Eq. (49) again, now from $r=r_{m}^{m e}$ to $r=r_{0}$, until the convergence criterion:

$$
\operatorname{det} \mathbf{z}^{m e}\left(r_{0}\right)=0
$$

is satisfied. This condition corresponds to the traction-free boundary condition at the outer layer.

The numerical algorithm used to solve the initial value problem is described in the Appendix.

\section{Finite element simulations in the post-buckling regime}

The linear stability analysis of Section 3 allows us to calculate the solution of the equilibrium problem (8) and (9) near the critical point, but does not allow us to study the evolution of the solution beyond the linear threshold. To explore the behavior of the system in the post-critical regime, we implement a user-defined material subroutine into the commercial finite element program Abaqus/Standard, Version 6.12 (Dassault Systemes Simulia, 2012). We model the growth using a pseudo-dynamic method similar to the one classically implemented for thermal dilatation. We adopt the following linear evolution law:

$$
g_{l}=1+\alpha_{l} \cdot t, \quad l=(e n, m e),
$$

where $\alpha_{l}$ are the growth rates for each layer, in our case $\alpha_{e n}=1$ and $\alpha_{m e}=0$, and $t$ is the time. We use an adaptive time stepping scheme with minimum and the maximum time steps of $t_{\max } \Delta=10^{-2}$ and $t_{\min } \Delta=10^{-3}$. We discretize the two-layered cylindrical model of Section 2 using tri-linear hybrid brick elements C3D8H in both layers. We use 800 elements in the circumferential direction, 12 elements in the radial direction, and 100 elements in the longitudinal direction. We choose the initial external radius to $R_{0}=1$ and systematically vary the parameters $H_{e n}, H_{m e}$, and $\mu_{m e} / \mu_{e n}$. Both layers of the cylinder are assumed to be Neo-Hookean and incompressible. To trigger the instability, we introduce an initial imperfection with an initial amplitude of $\varepsilon_{0}=0.001$ and a critical wavenumber as predicted by the linear stability analysis. In the following section we present the results of both the linear stability analysis according to Section 3 and the numerical simulation realized with Abaqus/Standard. 


\section{Results}

\subsection{Theoretical results of the linear stability analysis}

In this section, we present the analytical results from the linear stability analysis of Section 3. In particular, we investigate how the aspect ratios $H_{e n}$ and $H_{m e}$ of the endodermal and mesodermal layers and the stiffness ratio $\mu_{m e} / \mu_{e n}$ affect the onset of the instability and the morphology of the emerging pattern.

\subsubsection{Effect of the aspect ratios of the layers on the pattern selection}

Let us first investigate the effect that the geometric aspect ratios of the two layers have on the onset of the mechanical instabilities of the growing tube. Since the mesoderm is physiologically stiffer than the endoderm, we fixed the stiffness ratio to $\mu_{m} \mu_{e n}=5$ in Fig. 4 and $\mu_{m} \mu_{e n}=10$ in Fig. 5 . In both cases the initial external radius is $R_{0}=1$ and we vary the initial aspect ratio of the mesodermal layer $H_{m e}$.

In Figs. 4(a) and 5(a) we plot the critical growth ratio $\left(g_{e n} / g_{m e}\right)^{c r}$ against the initial aspect ratio of the endodermal layer $H_{e n}$ at different ratios of $H_{m e}$. The corresponding critical circumferential modes $m^{c r}$ and the critical longitudinal wave-numbers $k_{z}^{c r}$ are depicted in Figs. 4(b), 5(b) and Figs. 4(c), 5(c), respectively. The curves show how increasing either $H_{e n}$ or $H_{m e}$ decreases the critical growth ratio $\left(g_{e n} / g_{m e}\right)^{c r}$. A different trend can be observed in Fig. 4(b): the critical circumferential modes $m^{c r}$ increase as the initial aspect ratio $H_{m e}$ of the external layer increases, but the critical modes $m^{c r}$ decrease as the initial aspect ratio $H_{e n}$ of the internal layer increases. The critical longitudinal wavenumber $k_{z}^{c r}$, in Fig. $4(\mathrm{c})$, decreases as $H_{m e}$ increases. When the internal layer is thin, i.e., $H_{e n} \leq 1.3$, the following trends can be observed: low longitudinal wavenumbers emerge for cylinders with thick external layers, i.e., $H_{m e} \geq 2$, whereas high longitudinal wavenumbers emerge for cylinders with thinner external layer, i.e., $H_{m e} \leq 1.9$. In order to explore the behavior of the tissue when the mesodermal layer is thin, we fix the stiffness ratio to $\mu_{m d} \mu_{e n}=10$ and focus on the range $H_{\overline{m e}} 1.2-1.8$. In the following, we investigate the effect of the stiffness ratio between the two layers on the onset and the morphology of the instability.

\subsubsection{Effect of the stiffness ratio on the pattern selection}

Let us now fix the initial aspect ratio of the mesoderm $H_{m e}=1.8$ and the initial external radius $R_{0}=1$, and vary the stiffness ratio $\mu_{m e} / \mu_{\text {en }}$ between mesoderm and endoderm.

In Fig. 6(a) we plot the critical growth ratio $\left(g_{e n} / g_{m e}\right) r$ against the initial aspect ratio of the endodermal layer $H_{e n}$ at different stiffness ratios $\mu_{m e} / \mu_{e n}$. In Fig. 6(b) and (c) we illustrate the corresponding critical circumferential modes $m^{c r}$ and the longitudinal wavenumber $k_{z}^{c r}$, plotted against $H_{e n}$ at different $\mu_{m e} / \mu_{e n}$. Fig. 6(a) highlights how the critical growth factor $g_{e n} / g_{m e}$ decreases as the stiffness ratio $\mu_{m e} / \mu_{e n}$ increases: The stiffer the external layer, the lower the critical growth required to induce the instability. The curves in Fig. 6(b) and (c) show how an increase in the stiffness ratio corresponds to an

a

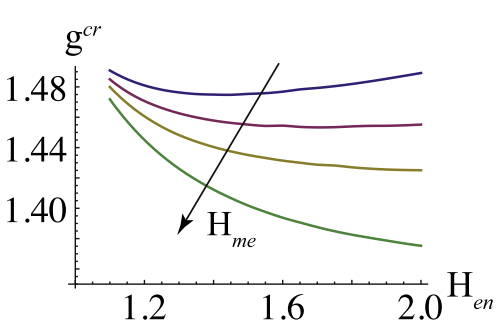

b

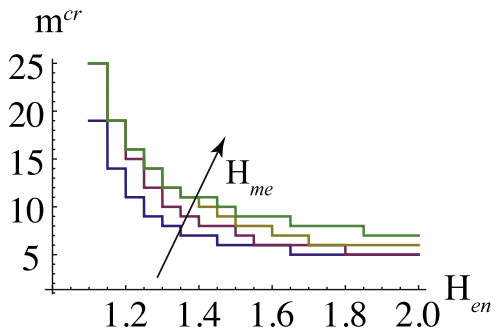

C

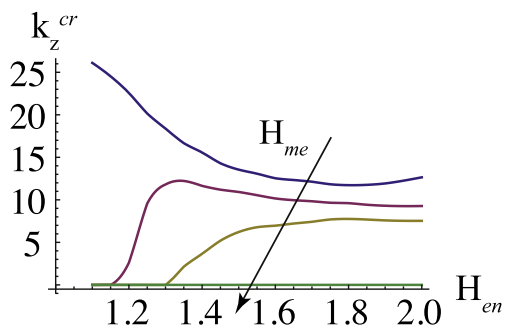

Fig. 4. Critical growth ratios $\left(g_{e n} / g_{m e}\right)^{c r}(a)$, with the corresponding critical circumferential modes $m^{c r}$ (b) and longitudinal modes $k_{z}^{c r}$ (c), plotted against the initial aspect ratio of the endoderm $H_{e n}$, at different initial aspect ratios of the mesoderm $H_{m e}=\{1.8,1.85,1.9,2\}$ for constant $\mu_{m e} / \mu_{\text {en }}=5$.
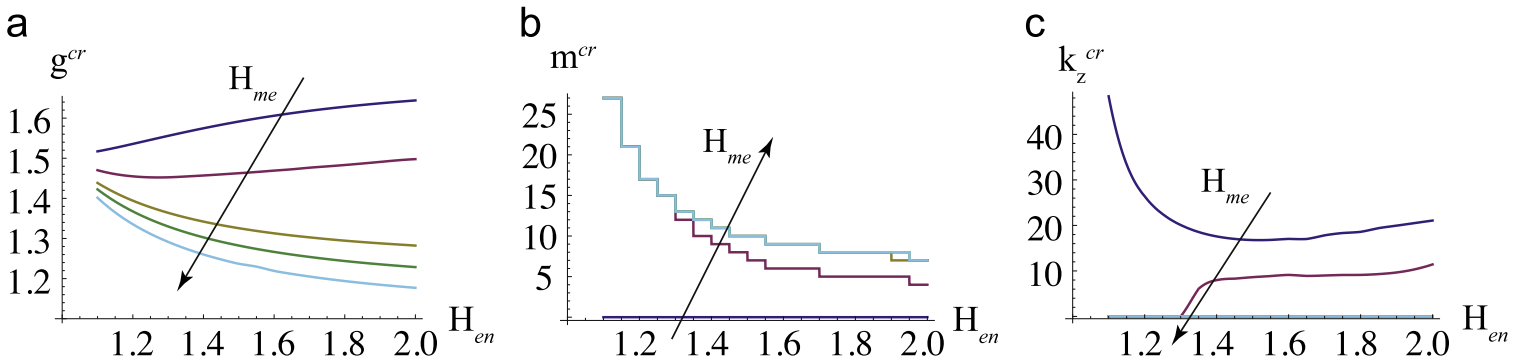

Fig. 5. Critical growth ratio $\left(g_{e n} / g_{m e}\right)^{c r}(a)$, with the corresponding critical circumferential and longitudinal modes $m^{c r}$ (b) and $k_{z}^{c r}$ (c), at different mesodermal aspect ratios $H_{m e}=1.2,1.3,1.4,1.5,1.8$, fixing $\mu_{m e} / \mu_{\text {en }}=10$. 
a

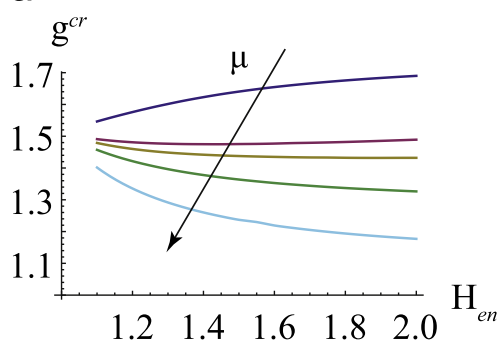

$\mathrm{b}$

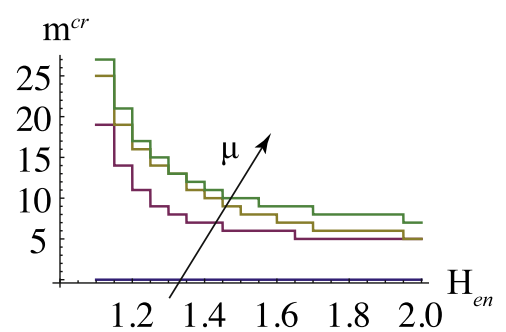

C

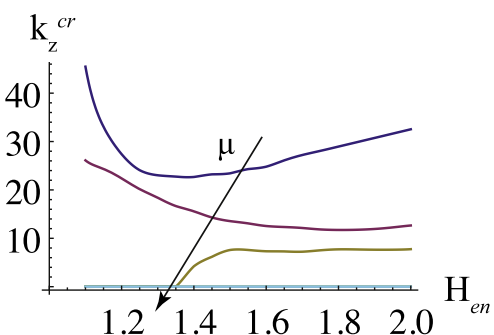

Fig. 6. Critical growth ratios $\left(g_{e n} / g_{m e}\right)^{c r}(a)$, with the corresponding critical circumferential modes $m^{c r}$ (b) and longitudinal modes $k_{z}^{c r}(c)$, plotted against the initial aspect ratio of the endoderm $H_{e n}$, at different stiffness ratios $\mu_{\text {me }} / \mu_{\text {en }}=\{3,5,5.3,6,10\}$ for constant $H_{m e}=1.8$.

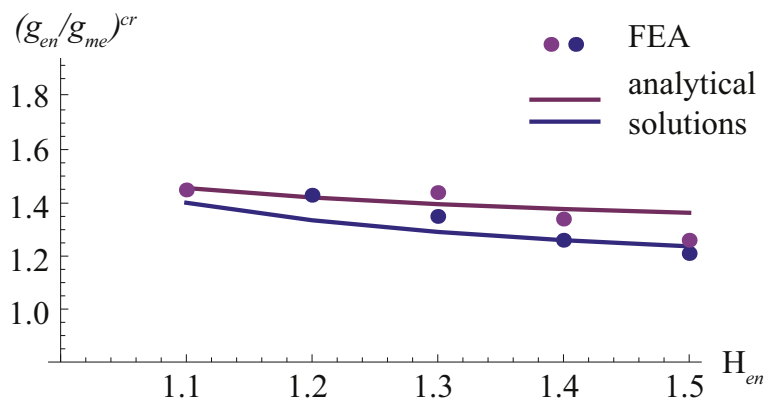

Fig. 7. Comparison between the critical growth value $\left(g_{e n} / g_{m e}\right)^{c r}$ from the numerical finite element solution (dots) and the analytical linear stability analysis (lines). The parameters are set as $H_{m e}=1.8, \mu_{m} \mu_{e n}=10$ (blue) and $\mu_{m} \downarrow \mu_{e n}=6$ (magenta). (For interpretation of the references to color in this figure caption, the reader is referred to the web version of this paper.)

increasing critical circumferential mode and a decreasing longitudinal wavenumber $k_{z}^{c r}$. In the following, we use the theoretical predictions either to validate the numerical code or to guide the numerical simulations in the post-buckling regime.

\subsection{Numerical results}

In this section we summarize the numerical results from the fully nonlinear finite element analysis performed with Abaqus/Standard. First, we validate the numerical implementation by comparing the theoretical predictions of Section 5.1 with the corresponding results from the numerical simulations. Second, we investigate the non-linear evolution of the instability patterns developing at the inner surface of the embryonic endoderm.

\subsubsection{Validation of the numerical code versus the theoretical predictions}

Let us first make an attempt to validate the finite element implementation by comparing the critical growth ratios in numerical simulation with the theoretical predictions. The criterion for such a comparison is based on the energy considerations. In this physical system the selected solution always minimizes the total elastic energy of the system. Before the onset of buckling, the solution with the minimal elastic energy is the basic axisymmetric deformation in Eq. (10). During the growth process, the stresses accumulate until a critical growth value is reached. At this point, the system bifurcates into a solution with a lower energy than the one associated with the basic axisymmetric solution, thus buckling occurs.

Here we identify the critical growth as the value of $\left(g_{e n} / g_{m e}\right)^{c r}$ at which the ratio between the total current elastic energy and the initial elastic energy $E_{\text {num }} / E_{0}$ has decreased by more than $1 \%$ of its initial value. The total elastic energy $E_{0}$ follows from integrating Eq. (5) as

$$
E_{0}=2 \pi\left(\int_{0}^{L} \int_{R i}^{R m} \psi_{e n} \cdot R_{e n} d R_{e n} d Z_{e n}+\int_{0}^{L} \int_{R m}^{R 0} \psi_{m e} \cdot R_{m e} d R_{m e} d Z_{m e}\right)
$$

where $\psi_{\mathrm{en}}$ and $\psi_{\mathrm{me}}$ are the strain energy functions of the endodermal and mesodermal layers associated with the basic deformation in Eq. (10).

In Fig. 7, we demonstrate that the critical growth values of our numerical simulations are in good agreement with the analytical predictions. The solid lines refer to the analytical solutions, whereas the markers indicate the numerical thresholds found from the simulations. This confirms the numerical implementation, which we will utilize in the following 
$\mathrm{H}_{m e}$

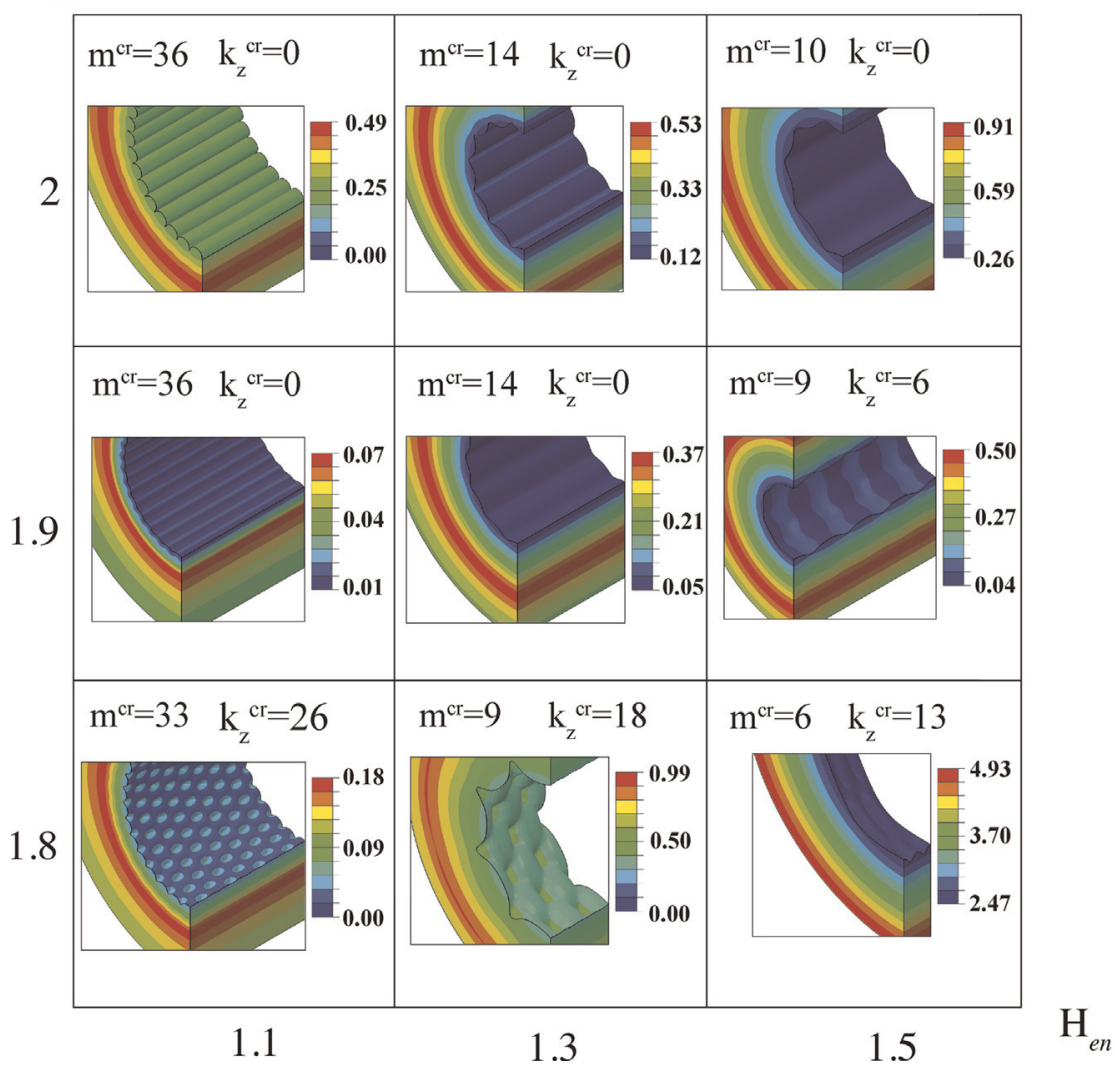

Fig. 8. Phase diagrams in the $\left(H_{e n}, H_{m e}\right)$ - space at constant $\mu_{m e} / \mu_{e n}=5$ : different instability patterns emerge during the numerical simulations, a onedimensional circumferential pattern for $m^{c r}>0$, and $k_{z}^{c r}=0$ and a bi-dimensional pattern for $m^{c r}>0$ and $k_{z}^{c r}>0$, where the colorbar indicates the value of the radial displacement.

to investigate pattern formation in the fully nonlinear regime.

\subsubsection{Evolution of the instability pattern}

Now, let us analyze the morphological evolution of the instability patterns far beyond the onset of buckling.

Fig. 8 collects the resulting patterns in the $\left.H_{e n}, H_{m e}\right)$ - space. The phase diagram in Fig. 8 highlights a transition from a onedimensional circumferential pattern to a bi-dimensional pattern, already indicated by the analytical curves in Fig. 4. In particular, tissues with both thick endoderm and mesoderm select a circumferential instability pattern with $m^{c r}>0$ and $k_{z}^{c r}=0$, whereas tissues with both thin endoderm and mesoderm select a bi-dimensional instability pattern with $m^{c r}, k_{z}^{c r}>0$.

Fig. 9 illustrates the phase diagram of the instability patterns in the $\left(H_{e n t} / \mu_{e n}\right)-$ space. In agreement with the analytical curves in Fig. 6, the instability pattern undergoes a transition from a one-dimensional to a bi-dimensional mode in Fig. 9. In particular, for high stiffness ratios $\mu_{m e} / \mu_{e n}$ a circumferential pattern with $m^{c r}>0$ and $k_{z}^{c r}=0 \quad$ is selected, whereas for low stiffness ratios $\mu_{m e} / \mu_{e n}$ a longitudinal pattern with $m^{c r}>0$ and $k_{z}^{c r}=0$ emerges. The region in which the shear moduli are of the same order is characterized by a bi-dimensional pattern with $m^{c r}>0$ and $k_{z}^{c r}>0$. In the next subsections, we characterize the fully nonlinear evolution for each of these endodermal patterns and investigate the amplitude of the emerging surface undulations.

One-dimensional circumferential pattern: Fig. 10 shows the amplitudes $\varepsilon_{\theta}$ of the one-dimensional circumferential instability pattern, $k_{z}^{c r}=0$, for varying growth factors $g_{\text {en }} / g_{m e}$. We normalize the amplitude with respect to the average internal radius $\bar{r}_{i}$ such that $\varepsilon_{\theta}=\left(r_{i}^{\max }-r_{i}^{\min }\right) / \bar{r}_{i}$, where $\bar{r}_{i}$ is the average of $r_{i}$ for each growth multiplier $g_{e n} / g_{m e}$ and $r_{i}^{\min }$ and $r_{i}^{\text {max }}$ are the minimum and maximum radii among all the values calculated for the nodes on the inner surface. Before the critical point is reached (1), the simulations show a slow increase in the amplitude of the perturbation. The instability thresholds of Section 5.1 are marked with crosses in Fig. 10. Beyond the critical point (2), the curves become steeper, reveal a fast increase in amplitude (3), then show a discontinuity which corresponds to the incipient formation of creases, whose width increases over increasing growth ratio (4-5).

One-dimensional longitudinal pattern: Fig. 11 illustrates the amplitudes $\varepsilon_{z}$ of the one-dimensional longitudinal instability pattern for different thicknesses of the internal layer $H_{e n}$. To calculate $\varepsilon_{z}=\left(r_{i}^{\max }-r_{i}^{\min }\right) / \bar{r}_{i}$, we calculate $\bar{r}_{i}, r_{i}^{\min }$, and $r_{i}^{\max }$ 


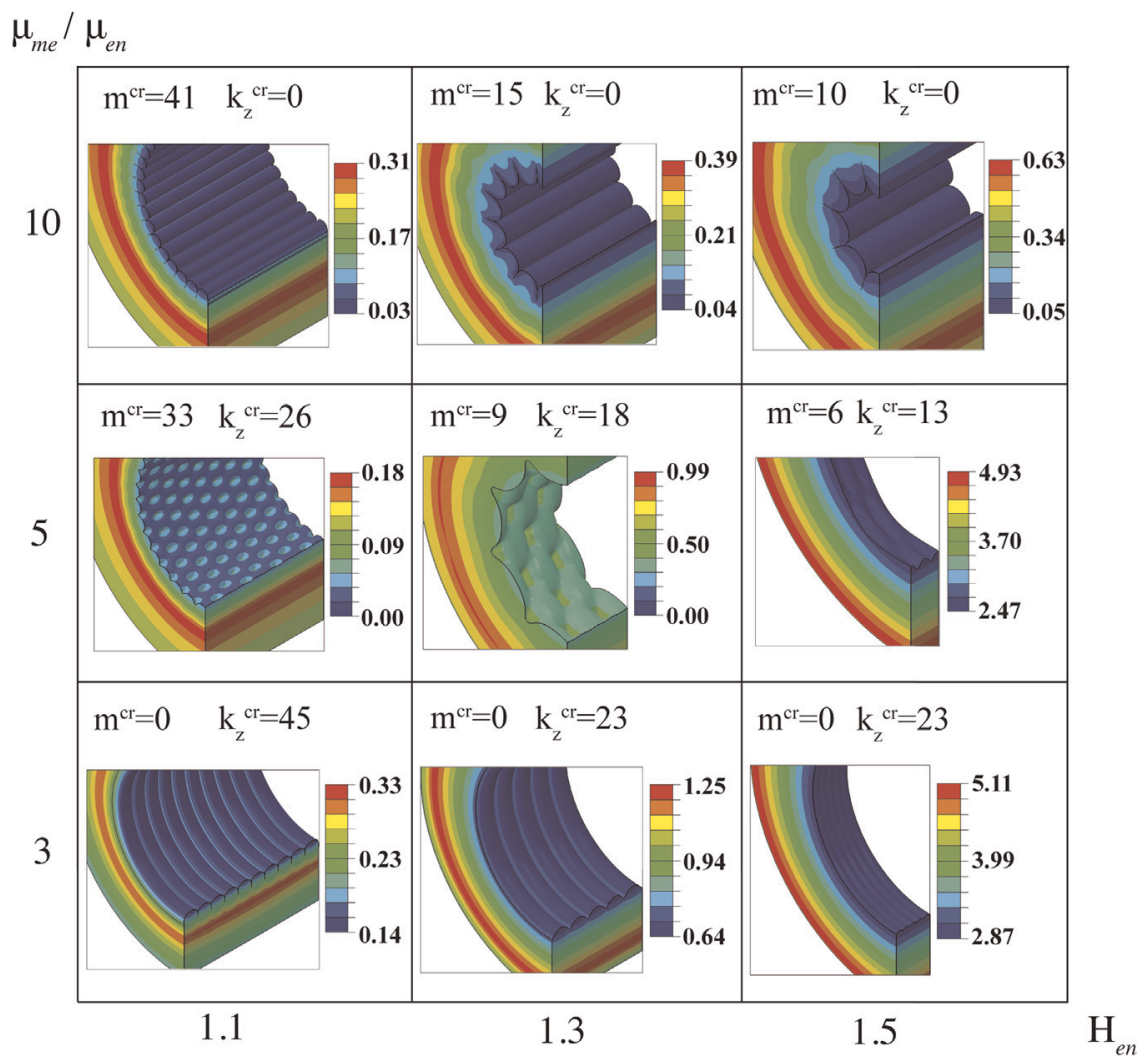

Fig. 9. Phase diagrams in the $\left(H_{e n}, \mu_{m e} / \mu_{e n}\right)$ - space at constant $H_{m e}=1.8$ : different instability patterns emerge during the numerical simulations, a onedimensional circumferential pattern for $m^{c r}>0$ and $k_{z}^{c r}=0$, a one-dimensional longitudinal pattern for $m^{c r}=0$ and $k_{z}^{c r}>0$, and a bi-dimensional pattern for $m^{c r}>0$ and $k_{z}^{c r}>0$. The colorbar indicates the value of the radial displacement. (For interpretation of the references to color in this figure caption, the reader is referred to the web version of this paper.)

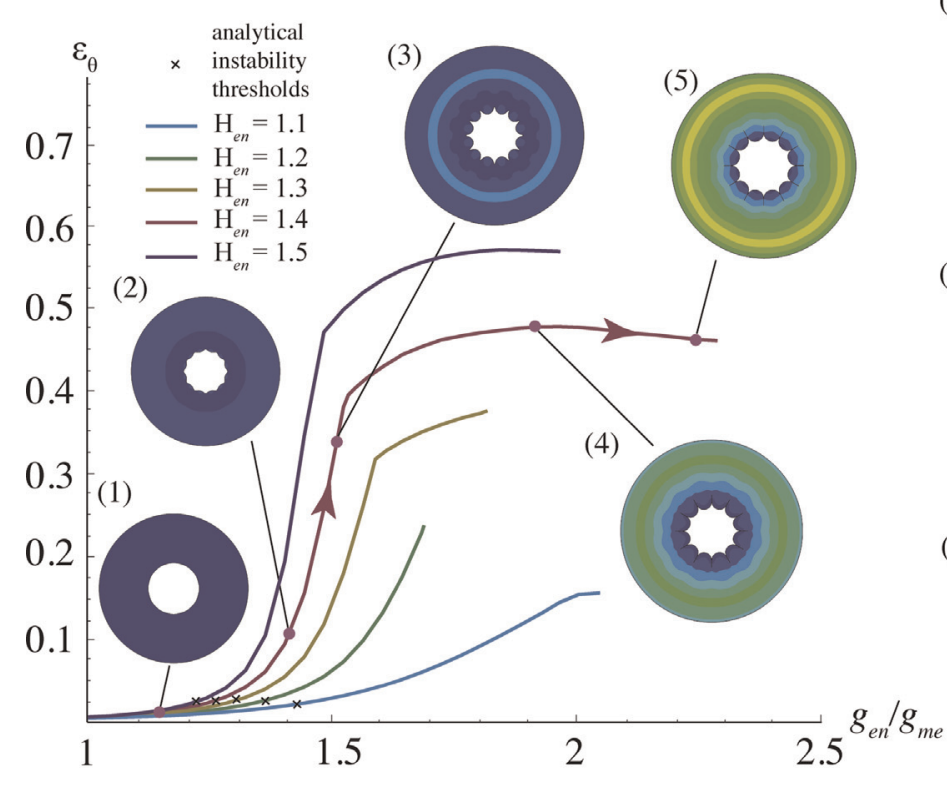

(1)

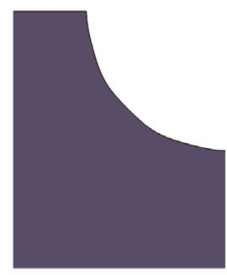

(2)

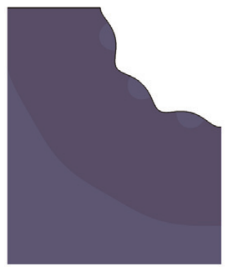

(3)

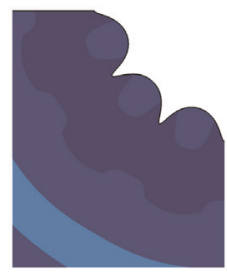

(4)

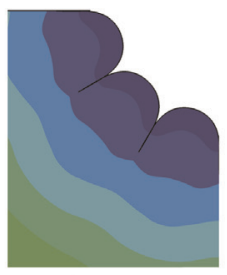

(5)

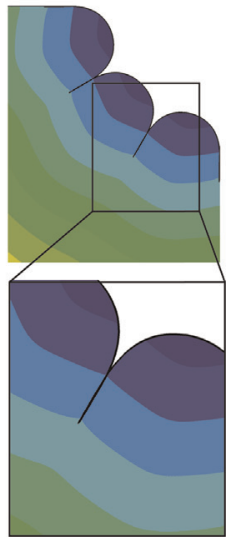

Fig. 10. (left) Amplitudes $\varepsilon \theta$ of the one-dimensional circumferential instability pattern, against the growth $g_{\text {en }} / g_{m e}$. The curves are shown for constant $H_{m e}=1.8$ and $\mu_{m e} / \mu_{e n}=10$ at varying $H_{e n}=\{1.1,1.2,1.3,1.4,1.5\}$. (right) Zoomed views of the resulting patterns depicted in the snapshots (1-5), highlighting the creasing of the inner surface. The colorcode indicates the absolute value of the radial displacement. (For interpretation of the references to color in this figure caption, the reader is referred to the web version of this paper.) 


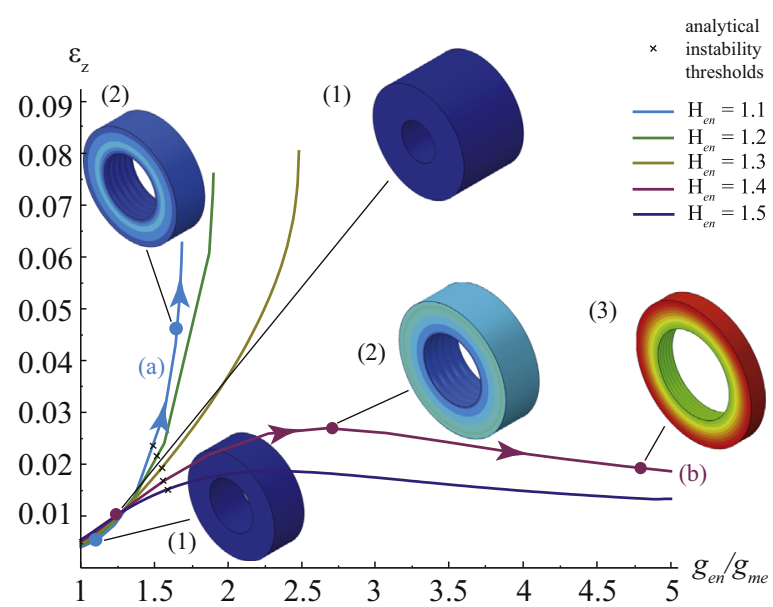

Fig. 11. Amplitudes $\varepsilon_{z}$ of the one-dimensional longitudinal instability pattern, against the growth $g_{\text {en }} / g_{m e}$. The curves are shown for constant $H_{m e}=1.8$ and $\mu_{\text {me }} / \mu_{\text {en }}=3$ at varying $H_{e n}=\{1.1,1.2,1.3,1.4,1.5\}$.
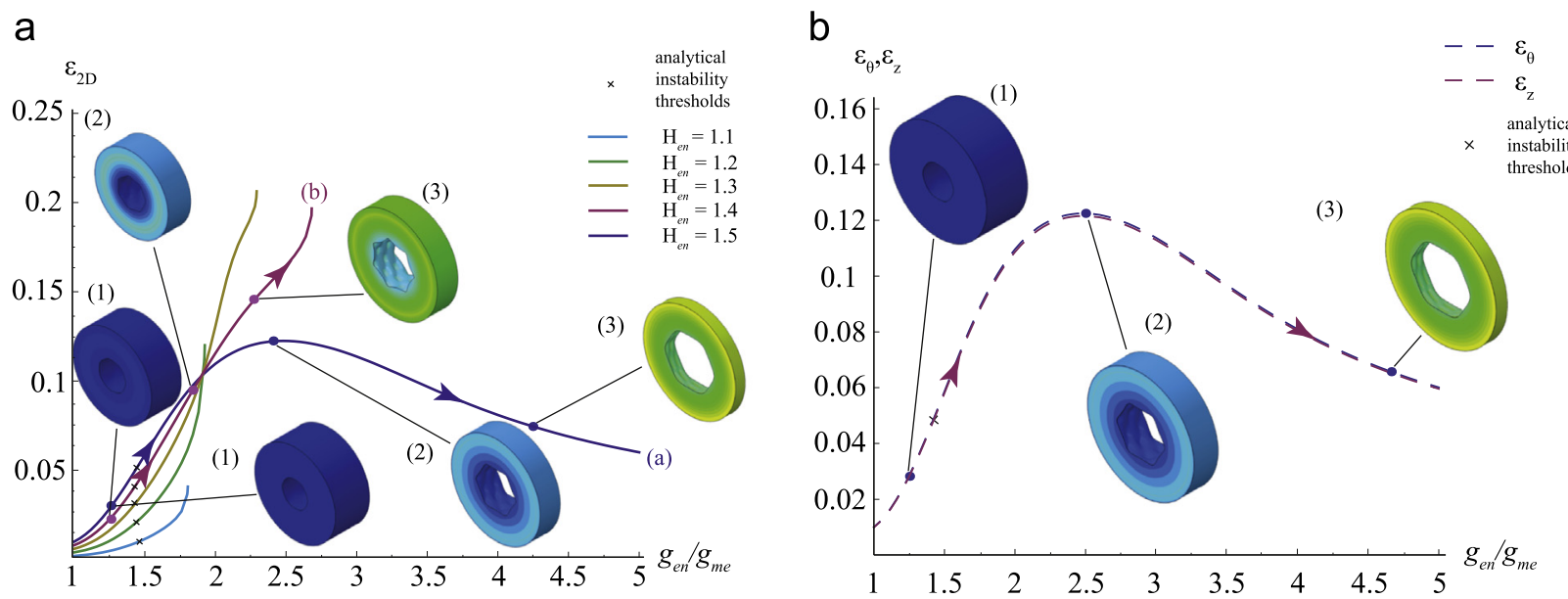

Fig. 12. (a) The amplitudes $\varepsilon_{22}$ of the bi-dimensional instability pattern, against the growth $g_{e n} / g_{m e}$. The curves are shown for constant $H_{m e}=1.8$ and $\mu_{m e} \mu_{e n}$ $=5$ at varying $H_{e n}=\{1.1,1.2,1.3,1.4,1.5\}$. (b) The two components $\varepsilon_{\theta}$ (blue) and $\varepsilon_{\mathrm{z}}$ (magenta) of the amplitude of the bi-dimensional instability pattern, against the growth $g_{e n} / g_{m e}$. The curves are shown for $\mu_{m e} \mu_{e n}=5, H_{e n}=1.5$ at $H_{m e}=1.8$ (dotted). (For interpretation of the references to color in this figure caption, the reader is referred to the web version of this paper.)

among all nodes of the inner surface, at a fixed $\theta$ coordinate. Two characteristic trends can be observed, as depicted by the lines $a$ and $b$ in Fig. 11, and the corresponding insets. Cylinders with a thinner inner layer, $H_{e n} \leq 1.3$, undergo a steep increase in the amplitude of the perturbation (line a) after the critical point, with a discontinuity, which indicates the formation of creases. Conversely, cylinders with a thicker inner layer, $H_{e n}>1.3$ (line b), undergo an initial increase in the wrinkling perturbation, followed by a gradual decrease over increasing growth. Therefore, the endoderm surface is characterized by stable wrinkles.

Bi-dimensional pattern: Fig. 12 depicts the amplitude of the bi-dimensional instability pattern. In Fig. 12(a), the value $\varepsilon_{2 D}=\left(r_{i}^{\max }-r_{i}^{\min }\right) / \bar{r}_{i}$ with $\bar{r}_{i}, r_{i}^{\min }$, and $r_{i}^{\max }$ is evaluated among all nodes at the inner surface. Similar to the one-dimensional longitudinal pattern, the curves now display two different trends: cylinders with a thicker inner layer, $H_{e n}>1.4$, select stable wrinkles with an initially increasing amplitude later reaching a saturation value (line a), whereas cylinders with a thinner inner layer, $H_{e n} \leq 1.4$, undergo a steep increase in the amplitude of the perturbation after the critical point, forming a creased surface. Moreover, both the circumferential $\varepsilon_{\theta}$ and the longitudinal $\varepsilon_{z}$ amplitudes of the bi-dimensional instability pattern have the same trend when plotted against the growth ratio $g_{e n} / g_{m e}$, as shown in Fig. 12(b).

\subsubsection{Secondary bifurcations: wavelength doubling}

The undulation wavelengths depicted in Figs. 10-12 remain unaltered for increasing values of $g_{e n} / g_{m e}$, so that the number of circumferential and longitudinal folds remains the same value selected by the linear stability analysis. Nonetheless, the numerical simulations highlight another possible scenario for the non-linear evolution of the instability. 


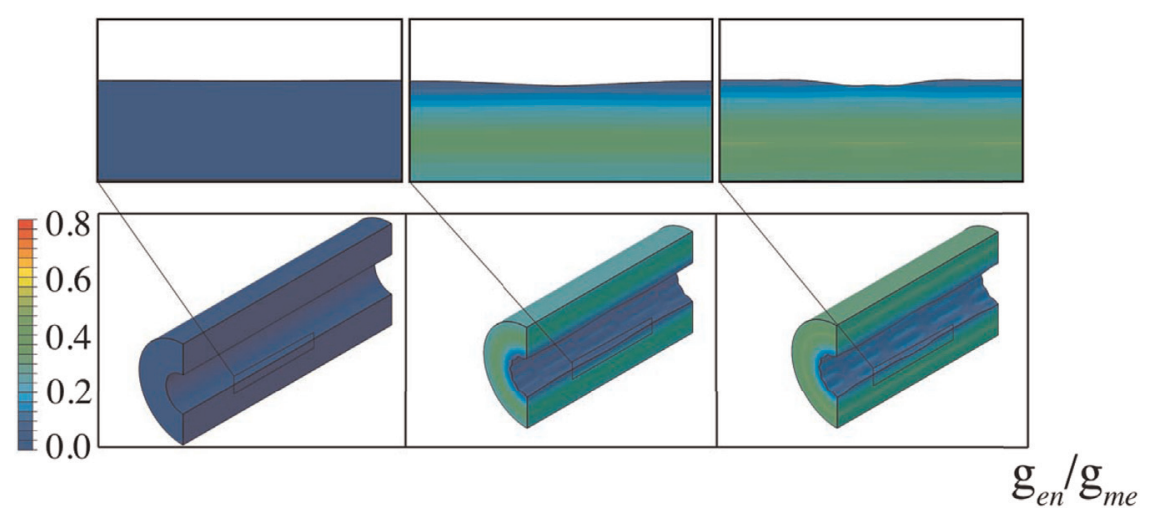

Fig. 13. Wavelength doubling of the bi-dimensional instability pattern, along the $z$-axes. The snapshots are taken at $g_{e n} / g_{m e}=\{1,1.72,1.81\}$ (left, middle, right), for $H_{e n}=1.35, H_{m e}=1.9$, and $\mu_{m} \mu_{e n}=5$. The colorbar indicates the value of the radial displacement. The predicted critical growth ratio is $\left.g_{e n} / g_{m e}\right)^{c r}=$ 1.44. (For interpretation of the references to color in this figure caption, the reader is referred to the web version of this paper.)

In Fig. 13, the instability initially begins with $m=12$ and $n_{z}=2$ as predicted by the analytical solution, but then, beyond the critical point $\left(g_{e n} / g_{m e}\right)^{c r}=1.44$, the number of longitudinal folds doubles. Therefore we observe a secondary transition from a square to a hexagonal surface pattern far beyond the linear stability threshold. Such a wavelength doubling is very similar to the one observed for the wrinkling of a stiff elastic layer over a soft substrate (Brau et al., 2011), undergoing a secondary bifurcation driven by the subharmonic resonance. A similar behavior has also been found for the circumferential folding of core-shell soft cylinders (Cao et al., 2012).

\section{Discussions}

In this work, we have established a morphoelastic model to explore the emergence of various endodermal structures in the embryonic gastrointestinal tissues of different vertebrate species. In Section 2, we modeled the embryonic gut as a twolayered cylinder with an inner layer representing the endodermal layer of the epithelium and the outer mesodermal layer, which includes both mesenchyme and muscular tissue prior to differentiation. Using the formalism of volumetric growth (Rodriguez et al., 1994), we modeled a different isotropic growth for both layers assuming a multiplicative decomposition of their deformation tensor. To provide a biologically relevant description of gastrointestinal organogenesis, we consider a stress-free growth of the intestinal tissue, which is considered for the first time here to be free of any spatial constraint. While more complex than homogeneous Dirichlet boundary conditions, homogeneous Neumann boundary conditions are more realistically represent the physiology of intestinal embryonic growth: as reported in Malas et al. (2003) and Sbarbati (1982), the external diameter of the embryonic gut increases with the embryonal age.

We assumed an isotropic, incompressible behavior for the embryonic gut layers, assuming a Neo-Hookean strain energy function to capture the mechanical non-linearity of the tissues. In Section 3, we performed a linear stability analysis with the aim of investigating the different instability patterns, which may emerge at the inner surface of the embryonic epithelium. First, we derived the basic axis-symmetric solution of the finite elastic problem. Second, we applied a small perturbation and solved the incremental boundary value problem. We calculated the critical differential growth ratio at the onset of the instability, and reported the associated circumferential and longitudinal modes for a wide range of geometric and mechanical parameters. Since the outer layer contains the developing muscular fibers, we particularly focused on a physiological range of values describing a mesoderm stiffer than the endoderm. We summarized the analytical results in Section 5.1. In Section 4, we performed nonlinear simulations in the post-buckling regime using a finite element model for finite volumetric growth. In Fig. 7, we validated the theoretical results by demonstrating an excellent qualitative and quantitative agreement between the predicted analytical and numerical growth thresholds. The numerical simulations allowed us to explore the fully nonlinear evolution of the emerging surface patterns. The corresponding morphological phase diagrams in the $\left.H_{e n}, H_{m e}\right)$ - and $\left(H_{e \not l}{ }_{e n} / \mu_{m e}\right)$ - spaces have been reported in Figs. 8 and 9.

Let us now discuss the results of this work in view of the few available data in the biological literature on intestine embryogenesis. From Fig. 4, we concluded that our model predicted the emergence of a circumferential pattern for embryonic intestinal tissues with thin epithelium and thick mesoderm. Conversely, a bi-dimensional pattern is selected for tissues with similar aspect ratios of the layers. Our results are in agreement with the experimental results from chick and mouse embryos reported in Shyer et al. (2013). In fact, circumferential folds emerge around day 8 after fertilization in chick embryos, which are characterized by a thin endoderm, whilst bi-dimensional patterns first arise in mouse embryos. In Fig. 14(a, b), we report the measurements of the area and external perimeter of the epithelium and mesenchyme of small and large intestine in mouse embryos (Sbarbati, 1982). Assuming a stress-free configuration at the first day and an isotropic growth process, we calculated the corresponding growth ratios for the small and large intestines at different embryonic ages, as depicted in Fig. 14(c). Our simulations indicate that the growth ratio for the small intestine is greater than the one 


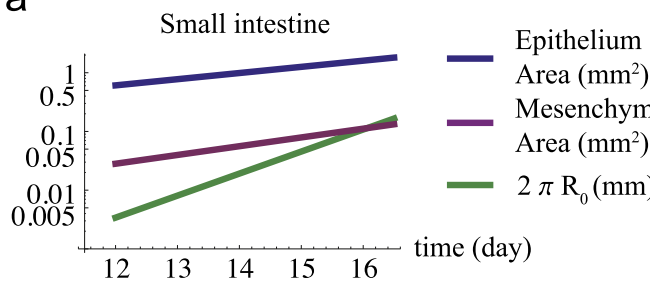

b
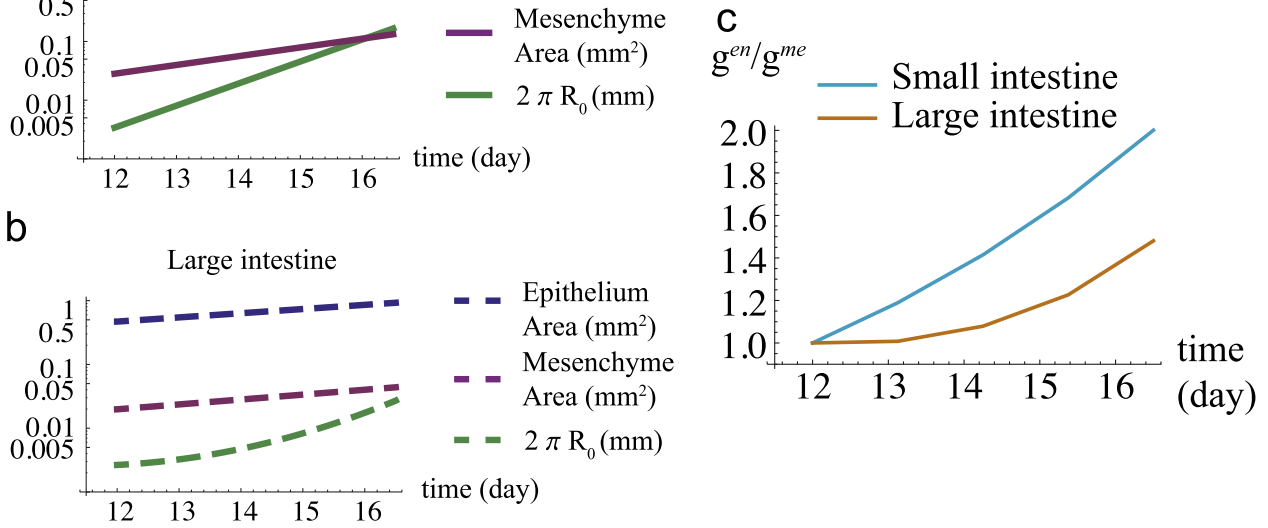

Fig. 14. Experimentally measured geometries of embryonic mouse intestines: outer circumference (blue), mesoderm area (magenta) and endoderm area (green) in the duodenum (a) and in the large intestine (b) (Sbarbati, 1982). Analytically predicted values of volumetric growth ratios for the small intestine (cyan) and large intestine (orange) over the days after fertilization (c). (For interpretation of the references to color in this figure caption, the reader is referred to the web version of this paper.)

Table 1

Geometrical parameters, thicknesses and diameter, of jejunum and ileum, ascending and descending colon, measured at the first trimester of gestation of a human fetus.

\begin{tabular}{llll}
\hline Geometrical parameter & Jejunum & Ileum & $\begin{array}{l}\text { Asc. } \\
\text { colon } \\
\text { colon }\end{array}$ \\
\hline Endoderm (mucosa) $(\mu \mathrm{m})$ & & 140 & 155 \\
$\begin{array}{l}\text { Mesoderm (muscular- } \\
\quad \text { is }+ \text { serosa) }(\mu \mathrm{m})\end{array}$ & 208 & 53 & 66 \\
$2 R_{0}(\mathrm{~mm})$ & 49 & 1 & 1.26 \\
$H_{\text {en }}$ & 1.5 & 1.45 & 1.38 \\
$H_{\text {me }}$ & 1.42 & 1.11 & 1.26 \\
\hline
\end{tabular}

for large intestine. In particular, a bi-dimensional pattern arises in the small intestine between day 13 and 14 after incubation at a growth factor $g_{e n} / g_{m e}=1.2-1.4$. This value is in agreement with the analytical predictions in Fig. 4: in fact, we can show that for an initial geometry with $H_{e n}=1.4, H_{m e}=2$ a bi-dimensional pattern occurs at a growth factor $g_{\text {en }} / g_{m e}=1.4$.

In Table 1, we report the geometrical measurements of the endodermal and mesodermal layers in different segments of the small and large intestines in human embryos (Malas et al., 2003). In the small intestine, where both the endoderm and the mesoderm are thick, villi start forming from a bi-dimensional pattern, while in the colon, characterized by a very thin mesoderm, a longitudinal pattern is observed first. This is in agreement with our analytical results, which predict the formation of longitudinal folds in the colon with a mesodermal aspect ratio in the range of $1.1 \underline{\underline{s}} H_{m e}<1.3$. In fact, the growth thresholds and the associated modes depicted in Fig. 5 show that a longitudinal folding pattern is selected for tissues with thin mesodermal layer $H_{m e}<1.3$.

Moreover, our results predict that intestines with a thicker endoderm have lower growth thresholds, which suggests that instabilities first occur in intestinal tissues with a thick endoderm. This finding is in qualitative agreement with the measurements, which showed that villi first form in the upper part of the duodenum where the epithelium is thicker with respect to the epithelium of the other gut segments in a human fetus (Johnson, 1910).

\section{Conclusion}

In conclusion, this work demonstrates that the elastic and the geometric parameters of the endoderm and the mesoderm can drive the formation of a wide range of patterns at the internal surface of embryonic guts. The onset of the instability is triggered by differential growth between the two layers and the emerging pattern can either take the form of creases or wrinkles in the fully nonlinear post-buckling regime. Although the theoretical and the numerical results are in good qualitative agreement with the biological literature, further biological measurements would be required to perform a more quantitative comparison. A current limitation of the proposed model is its specificity to isotropic growth processes, while 


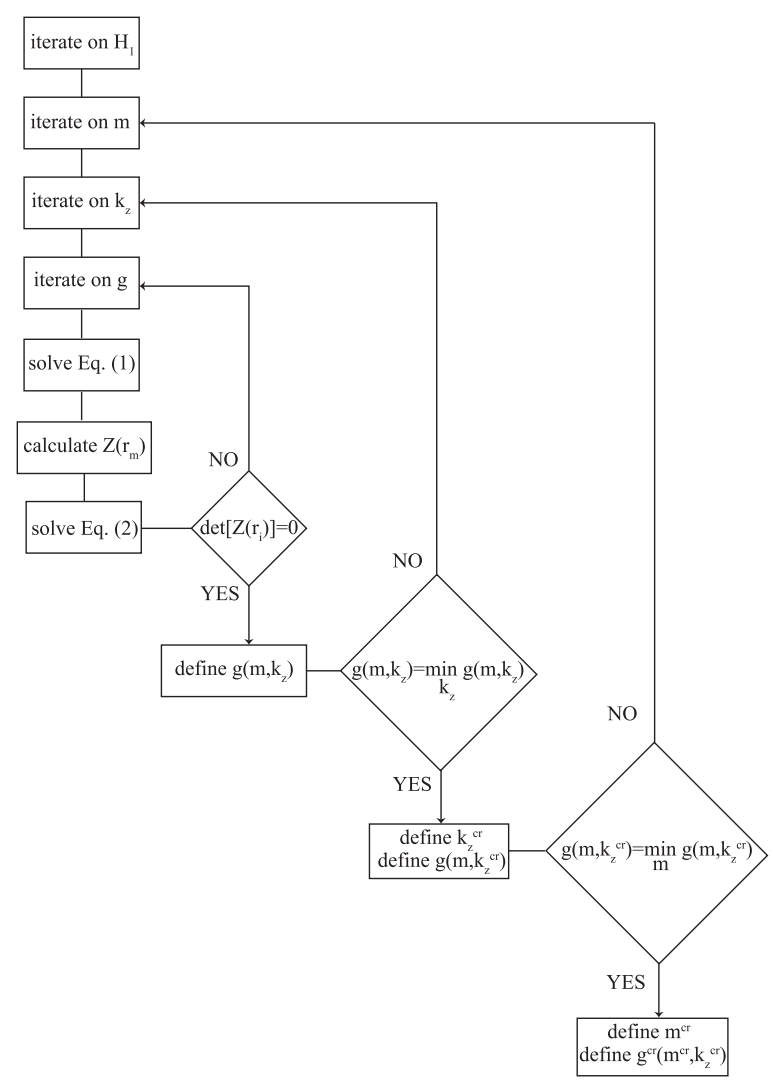

Fig. 15. Algorithmic flowchart to solve the differential Riccati equation (49).

anisotropic growth has been found to control the transition from circumferential patterns to the zigzag patterns in some vertebrate species. Future work will be devoted to explore the effects anisotropic differential growth and to develop suitable numerical algorithms for secondary bifurcations. Further refinements should also account for the presence of biochemical processes at different scales, which we have neglected in our current model. Typical phenomena of this kind including signaling mechanisms between epithelium and mesenchyme play a critical role in developmental sequence of tissue differentiation.

\section{Acknowledgment}

Partial funding from the France Stanford Center for Interdisciplinary Studies and the INSERM Grant OTPJ12U170 are gratefully acknowledged.

\section{Appendix A. Numerical algorithm}

In order to solve the initial value problem derived using the surface impedance method, we first iterate on $H_{e n}$ and then on the circumferential and longitudinal modes $m$ and $k_{z}$, until convergence. The critical growth factor $\left(g_{e n} / g_{m e}\right)^{c r}$ corresponds to the minimum value of $g_{e n} / g_{m e}$, which is a solution of Eqs. (49)-(52). The corresponding critical modes $m^{c r}$ and $k_{z}^{c r}$ are the circumferential and longitudinal modes associated with $\left(g_{e n} / g_{m e}\right)^{\text {cr }}$. Fig. 15 shows the flow chart of the numerical algorithm used to calculate the critical growth and the associated critical modes.

\section{References}

Balbi, V., Ciarletta, P., 2013. Morpho-elasticity of intestinal villi. J. R. Soc. Interface 10 (82), 20130109.

Ben Amar, M., Goriely, A., 2005. Growth and instability in elastic tissues. J. Mech. Phys. Solids 53 (10), $2284-2319$.

Ben Amar, M., Jia, F., 2013. Anisotropic growth shapes intestinal tissues during embryogenesis. Proc. Natl. Acad. Sci. 110 (26), $10525-10530$.

Biryukov, S.V., 1985. Impedance method in the theory of elastic surface waves. Sov. Phys. Acoust. 31, 350-354.

Brau, F., Vandeparre, H., Sabbah, A., Poulard, C., Boudaoud, A., Damman, P., 2011. Multiple-length-scale elastic instability mimics parametric resonance of 
nonlinear oscillators. Nat. Phys. 7 (1), 56-60.

Cao, Y.P., Li, B., Feng, X.Q., 2012. Surface wrinkling and folding of core-shell soft cylinders. Soft Matter 8 (2), 556-562.

Chadwick, P., 1997. The application of the Stroh formalism to prestressed elastic media. Math. Mech. Solids 2 (4), 379-403.

Ciarletta, P., Ben Amar, M., 2012a. Growth instabilities and folding in tubular organs: a variational method in non-linear elasticity. Int. J. Non-Linear Mech.

47 (2), 248-257.

Ciarletta, P., Ben Amar, M., 2012b. Pattern formation in fiber-reinforced tubular tissues: folding and segmentation during epithelial growth. J. Mech. Phys.

Solids 60 (3), 525-537.

Dassault Systemes Simulia. Abaqus 6.12 documentation. Providence, RI, US, 2012.

Destrade, M., Annaidh, A.N., Coman, C.D., 2009. Bending instabilities of soft biological tissues. Int. J. Solids Struct. 46 (25), $4322-4330$.

Eskandari, M., Pfaller, M.R., Kuhl, E., 2013. On the role of mechanics in chronic lung disease. Materials 6 (12), 5639-5658.

Fu, Y.B., 2007. Hamiltonian interpretation of the Stroh formalism in anisotropic elasticity. Proc. R. Soc. A: Math. Phys. Eng. Sci. 463 (2088), $3073-3087$.

Hannezo, E., Prost, J., Joanny, J.-F., 2011. Instabilities of monolayered epithelia: shape and structure of villi and crypts. Phys. Rev. Lett. 107 (7), 078104.

Haughton, D.M., Ogden, R.W., 1979. Bifurcation of inflated circular cylinders of elastic material under axial loading: ii. Exact theory for thick-walled tubes. J.

Mech. Phys. Solids 27 (5), 489-512.

Hilton, W.A., 1902. The morphology and development of intestinal folds and villi in vertebrates. Am. J. Anat. 1 (4), 459-505.

Johnson, F.P., 1910. The development of the mucous membrane of the oesophagus, stomach and small intestine in the human embryo. Am. J. Anat. 10 (1),

$521-575$.

Li, B., Cao, Y.P., Feng, X.Q., Gao, H., 2011. Surface wrinkling of mucosa induced by volumetric growththeory, simulation and experiment. J. Mech. Phys. Solids

59 (4), 758-774.

Li, B., Cao, Y.-P., Feng, X.-Q., Gao, H., 2012. Mechanics of morphological instabilities and surface wrinkling in soft materials: a review. Soft Matter 8 (21), $5728-5745$

Malas, M.A., Aslankoc, R., Ungor, B., Sulak, O., Candir, O., 2003. The development of jejunum and ileum during the fetal period. Early Hum. Dev. 74 (2), 109-124.

McLin, V.A., Henning, S.J., Jamrich, M., 2009. The role of the visceral mesoderm in the development of the gastrointestinal tract. Gastroenterology 136 (7), 2074-2091.

Moulton, D.E., Goriely, A., 2011. Circumferential buckling instability of a growing cylindrical tube. J. Mech. Phys. Solids 59 (3), 525-537.

Nelson, M.R., King, J.R., Jensen, O.E., 2013. Buckling of a growing tissue and the emergence of two-dimensional patterns. Math. Biosci. 246 (2), 229241. Norris, A.N., Shuvalov, A.L., 2010. Wave impedance matrices for cylindrically anisotropic radially inhomogeneous elastic solids. Q. J. Mech. Appl. Math. 63

(4), 401-435.

Ogden, R.W., 1997. Non-linear Elastic Deformations. Dover Publications 1997, NewYork and Ellis Horwood Chichester 1984.

Papastavrou, A., Steinmann, P., Kuhl, E., 2013. On the mechanics of continua with boundary energies and growing surfaces. J. Mech. Phys. Solids 61 (6), $1446-1463$.

Rodriguez, E.K., Hoger, A., McCulloch, A.D., 1994. Stress-dependent finite growth in soft elastic tissues. J. Biomech. 27 (4), $455-467$.

Sbarbati, R., 1982. Morphogenesis of the intestinal villi of the mouse embryo: chance and spatial necessity. J. Anat. 135 (Pt 3), 477.

Shyer, A.E., Tallinen, T., Nerurkar, N.L., Wei, Z., Gil, E.S., Kaplan, D.L., Tabin, C.J., Mahadevan, L., 2013. Villification: How the gut gets its villi. Science 342 (6155), 212-218.

Skalak, R., Zargaryan, S., Jain, R.K., Netti, P.A., Hoger, A., 1996. Compatibility and the genesis of residual stress by volumetric growthJ. Math. Biol. 34 (8) 889-914.

Stroh, A.N., 1962. Steady state problems in anisotropic elasticity. J. Math. Phys. 41 (2), 77-103.

Wilcox, C.M., Muñoz-Navas, M., Sung, J.J.Y., 2012. Atlas of clinical gastrointestinal endoscopy. Elsevier Health Sciences.

Yasugi, S., 1993. Role of epithelial-mesenchymal interactions in differentiation of epithelium of vertebrate digestive organs. Dev. Growth Differ. 35 (1), 1 9. 